\title{
CONOCIMIENTO Y MANEJO TRADICIONAL DE AgAVE KARWINSKII EN EL SUR DE MÉXICO
}

\section{Traditional KNOWLEdge AND management of AgAVE KaRWINSKII IN SOUTHERn MeXICO}

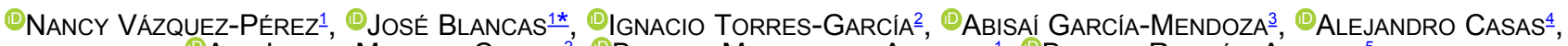

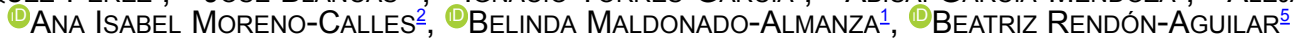 \\ ${ }^{1}$ Centro de Investigación en Biodiversidad y Conservación, Universidad Autónoma del Estado de Morelos, México. \\ ${ }^{2}$ Laboratorio de Estudios Transdisciplinarios Ambientales, Escuela Nacional de Estudios Superiores, Universidad Nacional \\ Autónoma de México, México. \\ 33ardín Botánico, Instituto de Biología, Universidad Nacional Autónoma de México, México. \\ ${ }^{4}$ Laboratorio de Manejo y Evolución de Recursos Genéticos, Instituto de Investigaciones en Ecosistemas y Sustentabilidad - \\ Universidad Nacional Autónoma de México, Campus Morelia, México. \\ ${ }^{5}$ Departamento de Biología - Universidad Autónoma Metropolitana-Iztapalapa. México.
}

*Autor de correspondencia: jose.blancas@uaem.mx

\section{Resumen}

Antecedentes: La intensidad de manejo en plantas está influida por factores socioecológicos, destacando el valor económico y la disponibilidad espacial de los recursos. Agave karwinskii es un recurso cultural, económica y ecológicamente relevante, con alta diversidad morfológica, la cual se refleja en la compleja nomenclatura tradicional. Algunos etnotaxones son mayormente valorados y enfrentan fuertes presiones debidas al auge en la demanda de mezcal.

Preguntas y/o hipótesis: ¿Cuáles etnotaxa de Agave karwinskii y con qué intensidad son manejados en su área de distribución? Los etnotaxa que son aprovechados para elaborar mezcales presentarán formas más intensas de manejo debido a su alto valor y demanda. Se identificarán distintas prácticas de manejo, desde las más incipientes hasta las más complejas, en función de su valor y disponibilidad.

Especie en estudio: Agave karwinskii Zucc.

Sitio de estudio y fechas: Puebla y Oaxaca, México. 2017-2018

Métodos: Se realizaron 43 entrevistas semiestructuradas a propietarios de fábricas de mezcal en el área de distribución. Se preguntó acerca del manejo de los distintos etnotaxa, prácticas de selección, atributos, valoración cultural y económica. Se calculó un índice de intensidad de manejo mediante un análisis de componentes principales.

Resultados: Se reconocieron 11 etnotaxones de A. karwinskii, principalmente para producir mezcal y como cerco vivo. Estos formaron tres grupos, correspondiendo a un gradiente de intensidad de manejo. Destacan aquellos que se propagan vegetativamente y se siembran por semilla en espacios antropogénicos.

Conclusiones: Se advierten procesos selectivos, los cuales pueden marcar rasgos de domesticación incipiente, como tamaños de roseta más grandes.

Palabras clave: Conservación in situ, domesticación incipiente, mezcal, sistemas agroforestales.

\footnotetext{
Abstract

Background: The intensity of management in plants is explained by socio-ecological factors, highlighting the economic value and the spatial availability of resources. Agave karwinskii is a culturally, economically, and ecologically relevant resource, with high morphological diversity, which is reflected in the complex traditional nomenclature. Some ethnotaxons are mostly valued and it faces strong pressures due to the boom in demand for mescal.

Question / Hypothesis: Which are the ethnotaxa of Agave karwinskii and with what intensity are they managed in the area of distribution of the species? Ethnotaxa that are used to produce mescal will present more intense forms of management due to their high value and demand. A broad spectrum of management practices will be identified, from the most incipient to the most complex, depending on their value and availability.

Studied species: Agave karwinskii Zucc.

Study site and years of study: Puebla and Oaxaca, Mexico. 2017-2018

Methods: 43 semi-structured interviews were conducted with owners of mescal factories in the distribution area. We asked about the management of different ethnotaxa, selection practices, morpho-physiological attributes, cultural and economic value of varieties. A management intensity index was calculated performing a principal component analysis.

Results: Eleven ethnotaxa of $A$. karwinskii were recognized, mainly used to produce mescal. These ethnotaxa formed three groups, corresponding to a gradient of management intensity, highlight those that have vegetative propagation and are sown by seeds in human-made areas.

Conclusions: The existence of selective processes is noted, which can mark features of incipient domestication, such as larger rosette sizes. Keywords: Agroforestry systems, incipient domestication, in situ conservation, mescal.
}

Este artículo se encuentra bajo los términos de la licencia Creative Commons Attribution License CCBY-NC (4.0) internacional. https://creativecommons.org/licenses/by-nc/4.0/ 
Las culturas tradicionales poseen modelos de percepción y apropiación de la naturaleza, los cuales se encuentran codificados en el Conocimiento Ecológico Tradicional (CET) (Beaucage 2012, Berkes \& Folke 1998, Toledo \& Barrera-Bassols 1984, 2008). Este se define como un cuerpo acumulativo de conocimientos acerca de las relaciones entre los seres vivos y su ambiente, el cual se transmite entre generaciones por mecanismos culturales, y es particularmente relevante en aquellas comunidades humanas que han tenido continuidad histórica en el tiempo y en el espacio (Berkes 1999, Brush 1993). Su estudio resulta crucial porque en este se condensan cientos e incluso miles de años de interacciones y experiencias prácticas de manejo de los recursos naturales, mismas que pueden aportar a la sostenibilidad (Boege 2008, Casas et al. 2016, Toledo \& Barrera-Bassols 2008), y replicarse en otros contextos por medio de su incorporación a las políticas públicas (Berkes 1999, Menzies 2006, Toledo \& BarreraBassols 2008).

Sin embargo, muchos de estos conocimientos se están erosionando (Saynes-Vásquez et al. 2013), y entre estos se incluyen técnicas de manejo de la diversidad vegetal (Martínez-Ballesté et al. 2006), los cuales se pierden debido a modificaciones en las formas y ritmos del manejo (Blancas et al. 2013), así como a cambios en los patrones de aprovechamiento (Beltrán-Rodríguez et al. 2017, HerschMartínez 1995, 1997, Torres et al. 2015a).

Esta situación está impactando en la sustentabilidad de los procesos de apropiación de la naturaleza y sus elementos, así como en el mantenimiento de la diversidad biológica en sitios silvestres, pero también de diversos agroecosistemas (Arnaiz-Schmitz et al. 2018, Drew \& Henne 2006, Moreno-Calles et al. 2016, Saynes-Vásquez et al. 2016, Tang \& Gavin 2016). Específicamente, el cambio de una dinámica de autoabasto a una regida por la demanda del mercado, ha marcado pautas en el manejo con resultados ambivalentes (Shackleton et al. 2015), con una tendencia a tener saldos negativos en términos de viabilidad de los recursos y la dinámica social que se ha generado (Lima-da Silva et al. 2019).

Se ha definido como manejo a todas aquellas intervenciones dirigidas a transformar o adaptar los ecosistemas, sus componentes o sus procesos de acuerdo con un plan humano (Blancas et al. 2013, Casas et al. 2016, González-Insuasti \& Caballero 2007). Conocer y estudiar en detalle las formas de manejo de los recursos naturales y los ecosistemas puede darnos elementos para entender la problemática que enfrentan, la dinámica socioecológica involucrada en sus distintas esferas (aprovechamiento, conservación, restauración y ordenamiento) (Casas et al. 2016) y escalas (local, regional, nacional y global) (Gunderson \& Holling 2002, Kates et al. 2001), así como para identificar y diseñar estrategias de manejo sustentable.
Asimismo, documentar el espectro de intensidades de manejo de los recursos bióticos, permite analizar la diversidad de prácticas y técnicas que posibilitaron la domesticación de plantas en el pasado (Casas et al. 2016, Casas et al. 1997a), así como los factores socioculturales y ecológicos que inciden en la emergencia de algunas especies de plantas como recursos clave en las actividades productivas de muchas comunidades rurales en el presente (Blancas et al. 2013, Hildebrand 2003, Rangel-Landa et al. 2017).

El manejo de los agaves en México constituye un foco de interés, ya que éstos son recursos ampliamente utilizados desde hace miles de años, en diversos entornos ecológicos y con una multiplicidad de propósitos (Colunga-GarcíaMarín \& May-Pat 1993, Colunga-GarcíaMarín et al. 1996, Colunga-GarcíaMarín et al. 2017, García-Mendoza \& Galván 1995). Los agaves son recursos cultural y económicamente muy valiosos para quienes hacen uso y manejo de éstos, y a su vez enfrentan una serie de problemáticas socio-ecológicas que requieren la participación de diversos esfuerzos y enfoques (ColungaGarcíaMarín \& May-Pat 1993, Colunga-GarcíaMarín \& Zizumbo-Villarreal 2006, Colunga-GarcíaMarín \& Zizumbo-Villareal 2007, Delgado-Lemus et al. 2014, FélixValdez et al. 2016, Torres et al. 2015a, Torres-García et al. 2019, Vargas-Ponce et al. 2007, Zizumbo-Villarreal et al. 2012).

De las 159 especies del género Agave en México (GarcíaMendoza et al. 2019), alrededor de 74 son reportadas como alimento y aproximadamente 50 (entre especies, complejos de especies y etnotaxones) se usan para elaborar bebidas destiladas en 26 estados de México (Colunga-GarcíaMarín \& Zizumbo-Villareal 2007, 2017, Figueredo-Urbina et al. 2017, Torres et al. 2015b, Vargas-Ponce et al. 2009). Específicamente, algunas especies poseen una gran diversidad de etnotaxa, los cuales se usan y manejan para diversos propósitos. El término etnotaxón se utiliza para determinar categorías o variedades tradicionales que reflejan las discontinuidades entre la misma o distintas entidades taxonómicas, aunque no necesariamente obedecen a principios filogenéticos o evolutivos de la clasificación linneana (Berlin 1992), reflejan la diversidad cultural en cuanto a usos y variaciones morfológicas. Es precisamente en especies de agaves con gran número de etnotaxones en las cuales reviste particular importancia documentar el manejo, ya que existe una gran presión por la creciente demanda y popularidad de algunos destilados, a fin de dar pautas para la sostenibilidad en su aprovechamiento.

En particular, el manejo de Agave karwinskii puede ejemplificar esta compleja dinámica de los sistemas socioecológicos, ya que es una especie que tiene una distribución relativamente restringida -en el Valle de Tehuacán (Puebla) y en los Valles Centrales y Sierra Sur de 
Oaxaca-, y está siendo aprovechada de forma intensiva, sobre todo en los últimos cinco años, debido al auge en la producción de mezcal (Vázquez-Pérez 2015). Esto ha tenido importantes consecuencias, en lo ecológico y en lo social, ya que la demanda de materia prima para los destilados está provocando extinciones locales en la vegetación natural de algunas variedades de esta especie, las cuales son preferidas para la producción y el consumo de mezcal (Vázquez-Pérez 2015).

Estudios previos en el área de distribución de A. karwinskii han documentado diversos etnotaxa de esta especie, todos ellos nombrados y reconocidos por atributos particulares (Vázquez-Pérez 2015). Aunque se desconocen aún los factores que han influido en tal diversificación, es factible suponer que el manejo de los etnotaxa deseados puede ser responsable de tal diversificación. Esto se ha observado en otras especies del género Agave, en donde el manejo ha conducido a la diversidad morfológica, fisiológica e incluso genética de este grupo de plantas (Casas et al. 2016, Colunga-GarcíaMarín et al. 1993, Colunga-GarcíaMarín \& May-Pat 1993, 1997, Figueredo et al. 2014). En particular, la intensificación en el manejo puede conducir a distintos grados de domesticación, y una forma de reconocerlos es mediante el desarrollo de síndromes de domesticación (Colunga-GarcíaMarín et al. 1996, 2017).

El manejo ha promovido la abundancia de algunos etnotaxa en respuesta a la demanda, esto estimula su fomento o cultivo en diversos agroecosistemas (milpas, potreros, huertos). En contraste, algunas prácticas tienen efectos negativos sobre las poblaciones de agaves, ejemplo de esto es el caso de la cosecha intensiva de todo el conjunto de individuos de una población, sin tomar en cuenta la edad o la talla para ser aprovechados, lo que repercute de forma negativa en la calidad del producto, así como en la capacidad de recuperación de las poblaciones bajo aprovechamiento (Delgado-Lemus et al. 2014, Torres et al. 2013, 2015a).

Dado que no existe información detallada acerca del manejo de Agave karwinskii, esta investigación pretende mostrar un panorama general acerca de los etnotaxa que son aprovechados para los distintos propósitos, poniendo especial énfasis en aquellos que son usados para la elaboración de mezcales, al ser esta la práctica que genera mayor presión sobre sus poblaciones y plantaciones. Se documentan los conocimientos y prácticas asociadas al manejo tradicional de A. karwinskii en su área de distribución natural, los distintos entornos de manejo y la intensidad con que son manejados dichos etnotaxa, con el propósito de inventariar técnicas probadas y procesos de innovación que pueden ser de gran utilidad para diseñar estrategias de manejo sustentable.
Partimos de la premisa de que, al igual que otros agaves en Mesoamérica, los distintos etnotaxa que forman el complejo Agave karwinskii están siendo manejados de forma diferencial en entornos diversos, desde los silvestres hasta los espacios humanizados con distintos grados de intensidad. Por lo que, aquellos etnotaxa que son aprovechados para elaborar mezcales presentarán formas más intensas de manejo debido a su alto valor y demanda. Asumimos que es factible identificar un amplio espectro de prácticas de manejo, desde las más incipientes hasta las más complejas, en función de su valor sensu lato y de su disponibilidad.

\section{Materiales y métodos}

Área de estudio. Se eligió una localidad de estudio en el Valle de Tehuacán, Puebla, tres en los Valles Centrales y dos en la Sierra Sur de Oaxaca (Figura 1). Con respecto a la caracterización socioeconómica de las localidades seleccionadas, todas son comunidades rurales con niveles altos de marginación, en donde la actividad económica principal es la agricultura de temporal, principalmente de maíz y frijol para autoconsumo. En menor medida, también se presentan algunos cultivos comerciales como el tomate, chile y limón (Apéndice 1). A excepción de Zapotitlán Salinas, Puebla, en todas las comunidades estudiadas, la actividad mezcalera es un eje importante de la economía local y también un fuerte componente de la identidad cultural. Otras actividades económicas lo constituyen la confección de objetos y artesanías de palma y carrizo, y el ecoturismo. El nivel de instrucción formal se circunscribe a los niveles básicos, por la poca cobertura educativa. Todas poseen un importante componente cultural indígena, sin embargo, el número de hablantes de idiomas locales ha decaído dramáticamente en los últimos 20 años (ver Apéndice 2).

Conocimiento local de A. karwinskii. Se efectuaron 48 entrevistas abiertas y 43 semiestructuradas (Bernard 2011; Martin 2004). Las primeras sirvieron para documentar el sistema de clasificación de las variedades locales de A. karwinskii, nomenclatura y categorías de uso. Las segundas permitieron identificar las estrategias y prácticas de manejo que les procuran, las razones que expone la gente para hacer tales prácticas, así como los espacios en donde se propagan, incluyendo el bosque y zonas manejadas.

La mayor parte de los colaboradores corresponden a maestros mezcaleros (personas que aprendieron el oficio de hacer mezcal de generación en generación y poseen conocimientos excepcionales de todo el proceso), quienes trabajan $\mathrm{y} / \mathrm{o}$ son propietarios de "palenques" (pequeñas fábricas artesanales de mezcal), ya que son los que poseen mayor conocimiento sobre el uso y manejo de esta especie. 


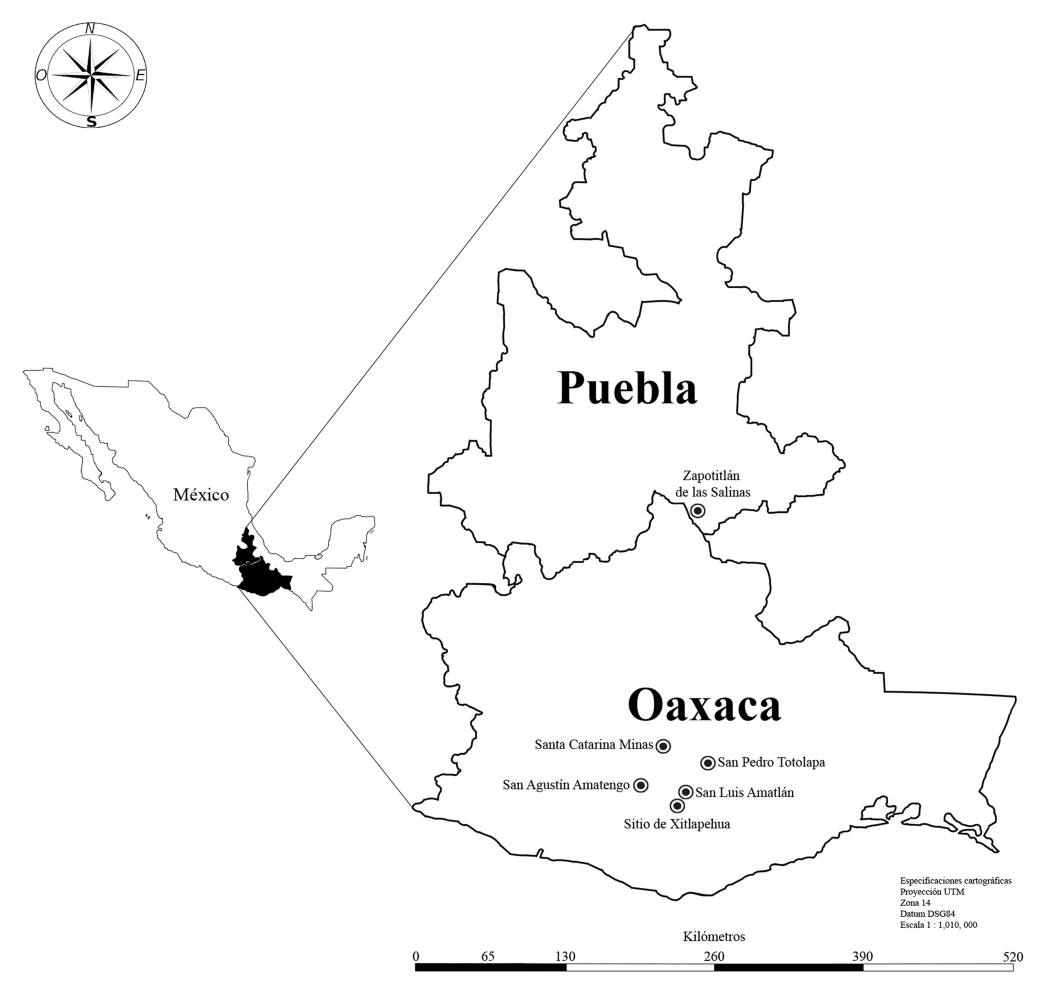

Figura 1. Ubicación geográfica de las áreas de estudio.

La muestra incluyó a 25 hombres y 18 mujeres, éstas últimas fueron amas de casa $\mathrm{y} / \mathrm{o}$ esposas de maestros mezcaleros de las diferentes comunidades. El número de personas entrevistadas por comunidad y el tipo de entrevista aplicada se indica en el Apéndice 2. La información se completó con notas de campo, fotografías, video y con la estrecha convivencia con los pobladores, por medio de observación participante (Martin 2004).

Análisis de la información y estimación del Índice de Intensidad de Manejo. Para documentar y analizar en detalle las formas de manejo que se les procuran a cada uno de los etnotaxones de A. karwinskii, así como la relación con los espacios en donde son promovidos, se construyó una matriz de datos, la cual incluyó once etnotaxa de A. karwinskii en las columnas, las cuales fueron clasificadas de acuerdo con 27 variables que fueron evaluadas. Entre las variables se consideraron aspectos ecológicos y socioculturales del manejo de esta especie y se ponderaron de acuerdo con la complejidad de las prácticas, tiempo invertido en estas, y el número de personas involucradas en dichas prácticas (Apéndice 3). Para categorizar las estrategias y prácticas de manejo se consideró la clasificación propuesta por Blancas et al. (2013).

A partir de esta base de datos, se realizó un análisis de componentes principales (ACP) mediante el programa NTSyS (Rohlf 2000), usando la matriz anteriormente mencionada. Los datos fueron estandarizados, a fin de reducir el efecto de las distintas escalas empleadas y que esto pudiera sub o sobreestimar el efecto de alguna variable. Se usó como factor de sustracción la media y como factor de división la desviación estándar. Se calculó una matriz de similitud usando el índice de correlación. Se calculó el porcentaje de la variación explicada por el modelo, así como la matriz de cargas, la cual indica las variables más importantes en cada uno de los componentes principales.

Se construyó un índice de intensidad de manejo, modificando el método desarrollado por González-Insuasti \& Caballero (2007). Este índice consideró aspectos socio ecológicos del manejo, tales como: número de usos, número de formas de manejo, complejidad en el uso de herramientas para la obtención y el procesamiento de los productos usados o aprovechados, labores de mantenimiento (tipo de actividades realizadas para asegurar su disponibilidad), criterios de selección y tipo de selección practicada, forma de reproducción, número de unidades de manejo en las que se encuentra presente (tanto en la vegetación silvestre como en ambientes antropogénicos) (Apéndice 3). Los registros del primer componente principal (CP1) constituyeron el Índice de intensidad de manejo, bajo el supuesto que el CP1 refleja la mayor proporción de variabilidad del modelo.

Colecta de material botánico. Para conocer la identidad taxonómica de los distintos etnotaxones de Agave karwinskii, se colectaron ejemplares botánicos, los cuales fueron identificados y depositados en el Herbario Nacional de México (MEXU). 


\section{Resultados}

Usos y partes útiles. Se documentaron siete usos principales, destacando por su frecuencia de mención: la producción de mezcal (82\%), cercos vivos (71\%), combustible $(58 \%)$ y alimento (36\%). En contraste, la obtención de fibras (24\%), construcción (18\%) y medicinal (9\%), fueron poco mencionados. En cuanto a las partes útiles, destaca el uso del tallo y bases foliares para la producción de mezcal $(90 \%)$ y la planta completa para cercos vivos $(100 \%)$. En menor proporción se utiliza el escapo como combustible ( $45 \%$ ), los botones florales como alimento $(20 \%)$ y las hojas para la obtención de fibras $(5 \%)$.

Nomenclatura local de los etnotaxa de Agave karwinskii. En la Tabla 1 se resume información sobre la nomenclatura local de los distintos etnotaxa de A. karwinskii, características, usos, partes útiles, formas de manejo, así como espacios en los que son manejados. Se documentaron once etnotaxones, los cuales se pueden diferenciar por el desarrollo o no de un tallo evidente, la forma y tamaño de las espinas, dientes en los márgenes y por la coloración de las hojas (Figura 2). De manera general, los etnotaxa Madrecuishe, San Martín y Tripón no muestran un tallo evidente, en contraste con el resto, en los cuales el tallo puede llegar a medir entre 1.0 a $1.5 \mathrm{~m}$.

En cuanto a la presencia de dientes en los márgenes de las hojas, los etnotaxa que los presentan de manera muy conspicua son Bicuishe, Espina negra y Sierrudo. En cuanto a la coloración de las hojas, se presenta un gradiente de intensidad, el cual va del verde claro en Cachitún y Cirial, verde azulado en Madrecuishe, hasta un color verde intenso en Bicuishe, Espina negra, Sierrudo y Marteño.

En este sentido, los pobladores reconocen y diferencian las características de cada maguey, de modo que han establecido una nomenclatura local para nombrar "magueyes largos" a Bicuishe, Tobasiche, Sierrudo, Cirial y Marteño (con tallos de 1 a $1.5 \mathrm{~m}$ ), y "barrilles" a San Martín, Tripón y Madrecuishe (tallos menores de $0.4 \mathrm{~m}$ ). Aunque también refirieron algunos etnotaxones como tipo "chino" y uno de Madrecuishe tipo "coyote". Sin embargo, no se pudo corroborar la identidad taxonómica de estos últimos.

Formas de manejo. a) Recolección. Los etnotaxa Cachitún, Cirial y Cuishe son recolectados de la vegetación silvestre y no se practican otras labores de mantenimiento, debido a que se asocian con poca productividad para la elaboración del mezcal, por la forma alargada de sus tallos. Aunque, en caso de escasez de materia prima, estos pueden ser cosechados. b) Tolerancia. Los etnotaxa Bicuishe, Espina negra, Sierrudo, Tobasiche y Madrecuishe se valoran ampliamente, por lo que se dejan en pie cuando se apertura la vegetación natural para terrenos de cultivo, ya que perciben que pueden aprovecharse en el futuro. c) Fomento. Se promueve la abundancia de los etnotaxa Bicuishe y Madrecuishe, ya que en la vegetación silvestre se trasplantan propágulos vegetativos in situ de las plantas madre. Presentan un gran desarrollo de la roseta, por lo que son altamente valorados, sobre todo Madrecuishe, ya que está asociado a una gran calidad y rendimiento superior en la obtención de mezcal. d) Fomento ex situ. Se documentó en Bicuishe, Espina negra, Sierrudo, Tobasiche, Madrecuishe, Marteño y Tripón, específicamente por trasplante de propágulos vegetativos de las áreas silvestres, para plantarse en forma de cercos vivos en solares y huertos. A excepción de Marteño y Tripón, que únicamente se encuentran como cerco vivo, en los demás etnotaxones se procuran prácticas como deshierbe, abono, riego y remoción de las hojas muertas. Estas prácticas se realizan con el fin de acortar los tiempos de crecimiento y producción del escapo floral. e) Cultivo por propagación vegetativa. Se documentó para San Martín, Madrecuishe y Tripón, principalmente sembrados en linderos dentro de las milpas. Estos etnotaxa son los que reciben mayores cuidados, a fin de permitir un mayor desarrollo de las hojas y las espinas. En el caso de Madrecuishe, se busca la mejora en las condiciones de crecimiento para obtener mayores tamaños de la roseta, importante en la productividad del mezcal. Algunas de estas prácticas buscan acelerar el crecimiento de las plantas y comprenden el deshierbe manual, poda de hojas secas, abonado con estiércol de chivo, riego con manguera en el solar o huerto, deshije (la separación de la planta madre) y trasplante de hijuelos a diversos agroecosistemas. f) Cultivo por semillas en vivero. Se registró en Sierrudo, Espina Negra, Marteño, Bicuishe y Madrecuishe, aunque es esporádico y de muy reciente práctica, ya que antes del auge del mezcal no se consideraba importante. Se asocia a estrategias de largo plazo, por lo que las plantas cultivadas por semilla tardan más tiempo en poder ser cosechadas, en contraste con las obtenidas por hijuelos vegetativos. Sin embargo, las personas refieren que han comenzado a cultivar por semilla desde hace cinco años, como una respuesta a la escasez de la materia prima, sobre todo de Madrecuishe, cuyo destilado es muy apreciado y demandado en ciudades como Oaxaca o Ciudad de México. Cabe resaltar que, aunque del etnotaxa Tripón se obtiene un destilado muy apreciado, no desarrolla semillas viables (Figura 3).

Espacios en donde son aprovechados y propagados los etnotaxa de A. karwinskii. Todos los etnotaxones son aprovechados en la vegetación silvestre, con excepción de Marteño, Tripón y San Martín. Estos mayormente se presentan en los distintos agroecosistemas, tales como los 
Tabla 1. Etnotaxones de A. karwinskii con su nomenclatura local, características distintivas, partes útiles, formas de manejo y sitios de propagación.

\begin{tabular}{|c|c|c|c|c|}
\hline Etnotaxones / características & Usos & Partes útiles & Formas de manejo & $\begin{array}{c}\text { Sitios de } \\
\text { propagación }\end{array}$ \\
\hline $\begin{array}{l}\text { Bicuishe / tallo evidente con hojas } \\
\text { secas }\end{array}$ & $\begin{array}{l}\text { Comestible, producción de } \\
\text { mezcal, fibras, combustible y } \\
\text { cercos vivos }\end{array}$ & $\begin{array}{l}\text { Botones florales } \\
\text { (vigas), tallo, hojas, } \\
\text { escapo y planta } \\
\text { completa }\end{array}$ & $\begin{array}{l}\text { Tolerancia y trasplante } \\
\text { de hijuelos }\end{array}$ & $\begin{array}{l}\text { Vegetación silvestre, } \\
\text { milpas, huertos y } \\
\text { casas }\end{array}$ \\
\hline Cachitún / en forma de candel & $\begin{array}{l}\text { Comestible, medicinal y } \\
\text { combustible }\end{array}$ & $\begin{array}{l}\text { Botones florales } \\
\text { (cacayas o vigas), } \\
\text { espinas, escapo }\end{array}$ & Recolección & Vegetación silvestre \\
\hline Cirial / tallo evidente sin hojas secas & $\begin{array}{l}\text { Ocasionalmente para producir } \\
\text { mezcal }\end{array}$ & Tallo & Recolección & Vegetación silvestre \\
\hline Cuishe / tallo corto sin hojas secas & $\begin{array}{l}\text { Ocasionalmente para producir } \\
\text { mezcal y combustible }\end{array}$ & Tallo y escapo & Recolección & Vegetación silvestre \\
\hline $\begin{array}{l}\text { Espina negra / maguey largo con } \\
\text { espinas gruesas }\end{array}$ & $\begin{array}{l}\text { Cercos vivos y } \\
\text { ocasionalmente para producir } \\
\text { mezcal }\end{array}$ & $\begin{array}{l}\text { Planta completa y } \\
\text { tallo }\end{array}$ & $\begin{array}{l}\text { Trasplante de hijuelos y } \\
\text { promoción }\end{array}$ & $\begin{array}{l}\text { Vegetación silvestre y } \\
\text { milpas }\end{array}$ \\
\hline $\begin{array}{l}\text { Madrecuishe / barril de color cenizo } \\
\text { y espinas gruesas }\end{array}$ & $\begin{array}{l}\text { Mayormente para producir } \\
\text { mezcal, comestible, cercos } \\
\text { vivos y combustible }\end{array}$ & $\begin{array}{l}\text { Tallo, botones } \\
\text { florales, planta } \\
\text { completa y escapo }\end{array}$ & $\begin{array}{l}\text { Recolección, tolerancia, } \\
\text { promoción, protección y } \\
\text { cultivo por hijuelos y } \\
\text { por semillas }\end{array}$ & $\begin{array}{l}\text { Vegetación silvestre, } \\
\text { milpas }\end{array}$ \\
\hline $\begin{array}{l}\text { Marteño / maguey largo y espinas } \\
\text { largas y delgadas }\end{array}$ & $\begin{array}{l}\text { Mayormente para producir } \\
\text { mezcal y cercos vivos }\end{array}$ & $\begin{array}{l}\text { Tallo y planta } \\
\text { completa }\end{array}$ & $\begin{array}{l}\text { Trasplante de } \\
\text { individuos y cultivo de } \\
\text { partes vegetativas }\end{array}$ & Milpas \\
\hline $\begin{array}{l}\text { San Martín / barril con muchas } \\
\text { hojas de color verde limón }\end{array}$ & Producción de mezcal & Tallo & $\begin{array}{l}\text { Tolerancia, promoción, } \\
\text { protección y cultivo }\end{array}$ & Huertos y milpas \\
\hline $\begin{array}{l}\text { Sierrudo / maguey largo con } \\
\text { abundantes espinas gruesas }\end{array}$ & $\begin{array}{l}\text { Cercos vivos, fibras y } \\
\text { ocasionalmente para producir } \\
\text { mezcal }\end{array}$ & $\begin{array}{l}\text { Planta completa, } \\
\text { hojas y tallo }\end{array}$ & $\begin{array}{l}\text { Recolección, promoción } \\
\text { y trasplante }\end{array}$ & $\begin{array}{l}\text { Vegetación silvestre, } \\
\text { huertos, milpas y } \\
\text { casas }\end{array}$ \\
\hline $\begin{array}{l}\text { Tobasiche / maguey largo con hojas } \\
\text { en forma de cuchara y espinas } \\
\text { gruesas }\end{array}$ & $\begin{array}{l}\text { Cercos vivos, combustible y } \\
\text { ocasionalmente para producir } \\
\text { mezcal }\end{array}$ & $\begin{array}{l}\text { Planta completa, } \\
\text { escapo y tallo }\end{array}$ & $\begin{array}{l}\text { Trasplante de hijuelos, } \\
\text { fomento }\end{array}$ & $\begin{array}{l}\text { Vegetación silvestre, } \\
\text { huertos, milpas y } \\
\text { casas }\end{array}$ \\
\hline $\begin{array}{l}\text { Tripón / barril con hijuelos que } \\
\text { crecen en formade espiral }\end{array}$ & Producción de mezcal & Tallo & $\begin{array}{l}\text { Tolerancia, fomento y } \\
\text { trasplante de hijuelos }\end{array}$ & $\begin{array}{l}\text { Vegetación silvestre, } \\
\text { huertos, milpas y } \\
\text { casas }\end{array}$ \\
\hline
\end{tabular}

huertos familiares, casi siempre como cercos vivos, como en el caso de Marteño. Los etnotaxa Cachitún, Cuishe y Cirial sólo se encuentran y aprovechan en la vegetación silvestre. Por el contrario, Sierrudo, Espina negra, Tobasiche, Bicuishe y Madrecuishe, además de que se aprovechan en la vegetación silvestre, también se presentan en los agroecosistemas como cercos vivos y en linderos en el caso de Madrcuishe (Tabla 1).

Intensidad de manejo de los etnotaxa de A. karwinskii. En el análisis de componentes principales (ACP), los dos primeros componentes explican el $71.77 \%$ de la variación, y en particular el primer componente explica el 52.31\%. En la Figura 4 se observan los tres grupos que se obtuvieron en el ACP de acuerdo con la intensidad de manejo de los distintos etnotaxones. El primer grupo (círculo morado) lo constituyen Cachitún, Cuishe y Cirial, que no reciben labores de mantenimiento y cuya estrategia de manejo se limita a la recolección simple en áreas de vegetación silvestre. El uso de herramientas para su manejo no implica un grado de especialización, ya que estas se circunscriben a las propias de las labores agrícolas. Además, la existencia 

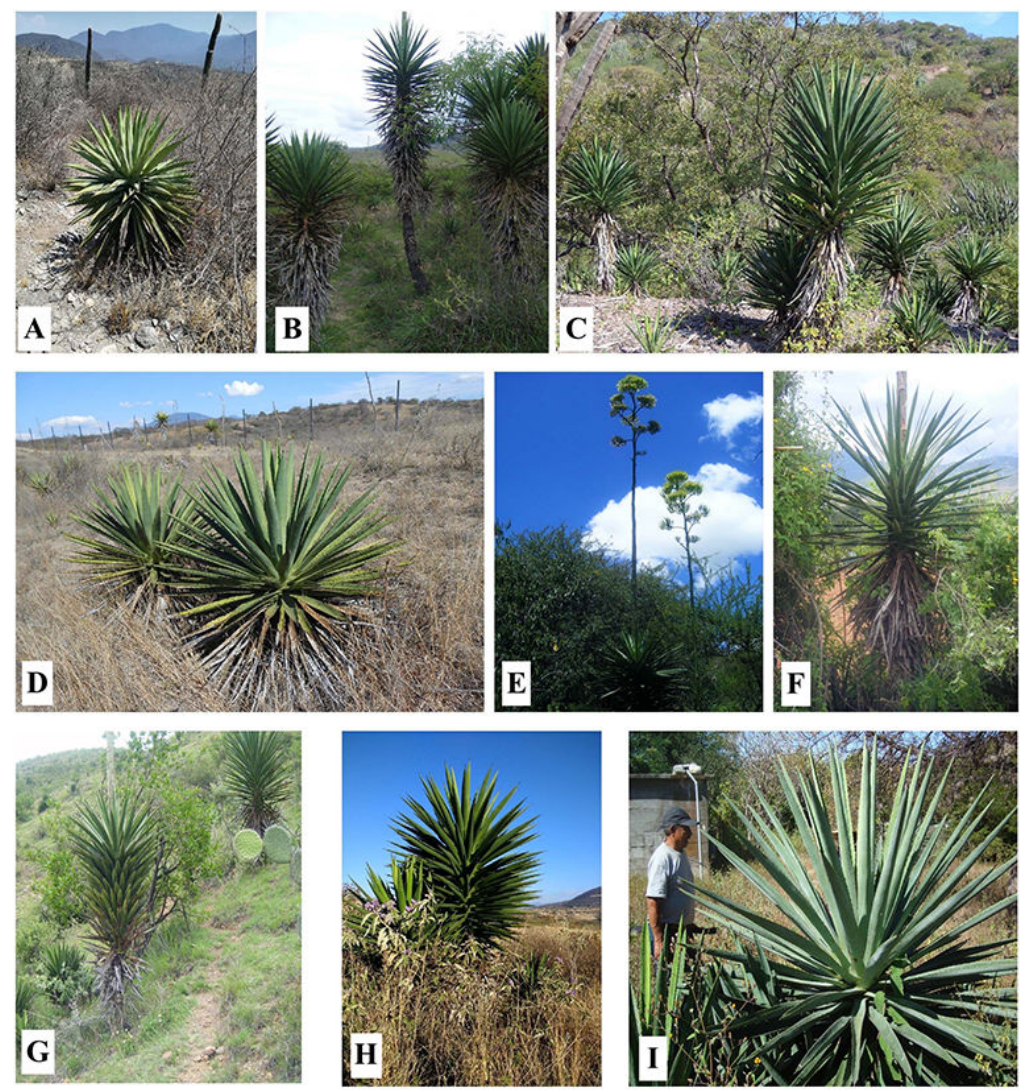

Figura 2. Algunos de los distintos etnotaxones de Agave karwinskii documentados en la zona de estudio y principales características que refieren las personas para diferenciarlos: A) Cachitún (en forma de candel), B) Cirial (tallo evidente sin hojas secas), C) Cuishe / (tallo corto sin hojas secas), D) San Martín (barril con muchas hojas de color verde limón), E) Espina negra (maguey largo con espinas gruesas), F) Marteño (maguey largo y espinas largas y delgadas), G) Sierrudo (maguey largo con abundantes espinas gruesas), H) Bicuishe (tallo evidente con hojas secas), I) Madrecuishe (barril de color cenizo y espinas gruesas) (Fotos: Nancy Vázquez Pérez).

de regulación o normas de acceso comunitario al recurso es inoperante, ya que, aunque existen multas cuando se cortan magueyes sin permiso, estas raramente se aplican.

El segundo grupo (círculo rojo) lo conforman San Martín, Espina negra, Tripón, Marteño, Tobasiche y Sierrudo, y se caracterizan por recibir deshierbe, remoción de hojas secas, deshije (remover propágulos vegetativos y trasplantarlos a nuevas áreas), ablandamiento del suelo, e incluso en algunos casos riego. Todas estas labores se realizan en contextos de proximidad a los huertos y parcelas de cultivo, y en menor medida en la vegetación silvestre. El uso de herramientas e insumos es más amplio que en el grupo anterior, no necesariamente por el manejo de las plantas en el lugar donde crecen, sino por el proceso para la obtención del mezcal. De ahí que, además de las herramientas propias de las labores agrícolas, se usan otros implementos como alquiler de animales de tiro para la molienda de los tallos cocidos, recipientes para la fermentación, leña para la destilación, etc. Las normas para acceder a estos recursos son inexistentes, ya que la mayoría de los individuos de estos etnotaxones se encuentran en parcelas de cultivo y huertos, en donde los manejadores deciden tolerarlos o eliminarlos en función de sus intereses, sin necesidad de consultar a ninguna autoridad.

El tercer grupo (círculo verde), lo constituyen Bicuishe y Madrecuishe, que al ser los preferidos en la producción de mezcal, son los que reciben el mayor número de labores de mantenimiento (Figura 5). Destacan, el deshije, deshierbe, riego y aplicación de insumos para evitar el ataque de insectos. Estas labores se realizan sobre todo en Madrecuishe, que se encuentra en parcelas de cultivo, formando pequeños linderos y es propagado por medio de trasplante de hijuelos. Ambos etnotaxones también pueden ser propagados por siembra de semillas. En cuanto al uso de herramientas para el manejo y a la complejidad de éstas, destacan las propias para las labores agrícolas, además de las necesarias para el procesamiento y obtención del mezcal.

Un número considerable de maestros mezcaleros mantienen el uso de herramientas tradicionales, como los 

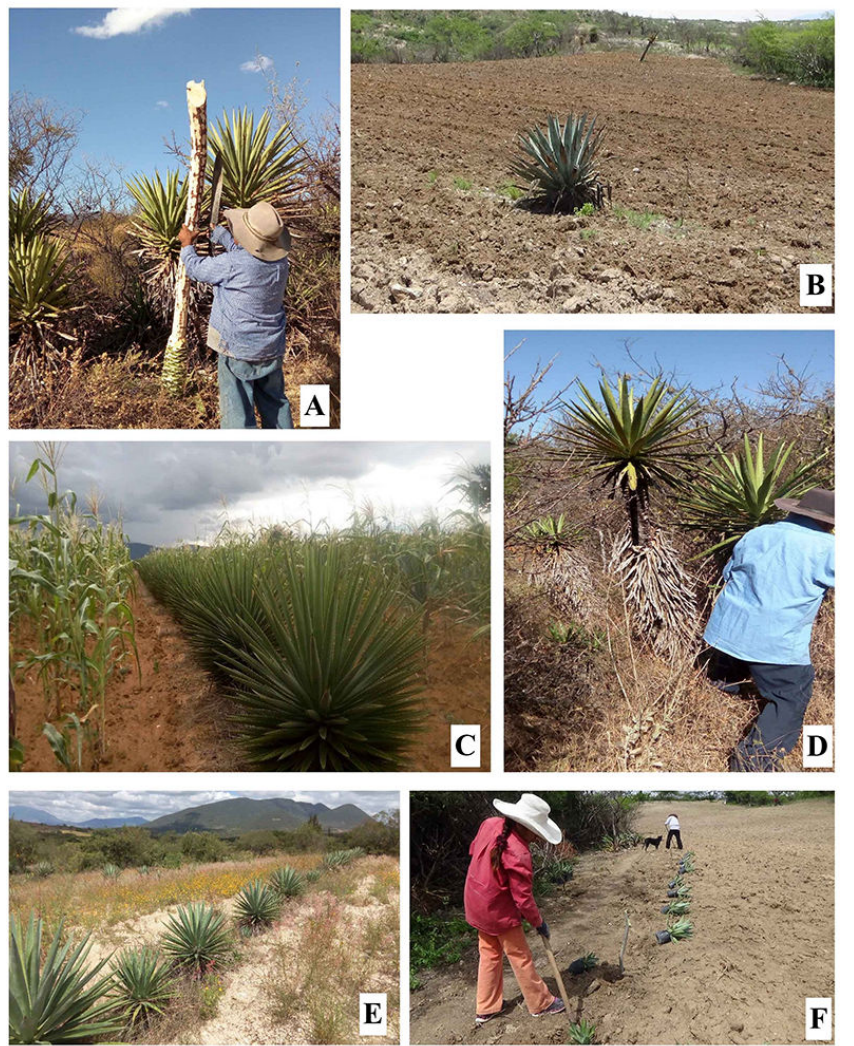

Figura 3. Estrategias de manejo documentadas para Agave karwinskii en la zona de estudio: A) recolección de Bicuishe en la vegetación silvestre; B) Tolerancia de Madrecuishe en parcelas de cultivo; C) Cultivo de San Martín por propagación de hijuelos en milpas; D) Protección de Tobasiche por medio de poda de hojas muertas; E) Cultivo de Madrecuishe por propagación de hijuelos; y F) Trasplante de individuos de Madrecuishe cultivados por semilla. (Fotos: Nancy Vázquez-Pérez).

mazos de madera, los cuales se ocupan en el proceso de molienda una vez cocido el maguey. Sin embargo, debido al auge mezcalero y a la alta demanda del mercado, muchas técnicas que emplean herramientas tradicionales se han ido perdiendo.

La diferencia en el manejo de estos etnotaxones con respecto a los del segundo grupo, es la intensidad y la frecuencia con que se utilizan. Bicuishe y Madrecuishe son promovidos o fomentados dentro de la vegetación silvestre a diferencia de los otros, en donde no se llevan a cabo tales estrategias. En Madrecuishe y Bicuishe se documentaron casi todas las categorías de manejo y esto puede estar relacionado con que ambos son altamente apreciados por las cualidades organolépticas del mezcal. En cuanto a la existencia de regulación o normas de acceso comunitario para acceder al recurso, en estos etnotaxones no se presenta, ya que la mayor parte de los individuos se encuentran en áreas en donde la propiedad está bien definida. Por lo tanto, la normatividad no opera en estos espacios, ya que esta se refiere a los espacios de uso común.

En cuanto al índice de intensidad de manejo, los registros del primer componente principal, muestran a Madrecuishe como el etnotaxón más intensamente manejado. En contraste, Cachitún es el menos intensamente manejado. Estos dos etnotaxones serían los extremos de un gradiente de intensidad de manejo, en donde también se encontrarían los nueve restantes (Tabla 2).

De acuerdo con la matriz de cargas (Tabla 3), las variables más importantes en el componente principal 1 (CP1) son: número de formas de manejo distintas que se les procuran, número de personas que las propagan por medio de hijuelos, número de personas que las promueven, número de labores de mantenimiento que reciben, número de personas que las protegen y la proximidad de los sitios de colecta (más de $5 \mathrm{~km}$ ). A su vez, el componente principal 2 (CP2) separa a los etnotaxones del círculo rojo del resto. Las variables más importantes en este componente fueron: la presencia de los etnotaxa en zonas silvestres, el número de personas que los recolectan y la proximidad de hasta 1 $\mathrm{km}$ a los sitios de colecta.

\section{Discusión}

La compleja nomenclatura con que se designan los distintos etnotaxones de $A$. karwinskii refleja el 


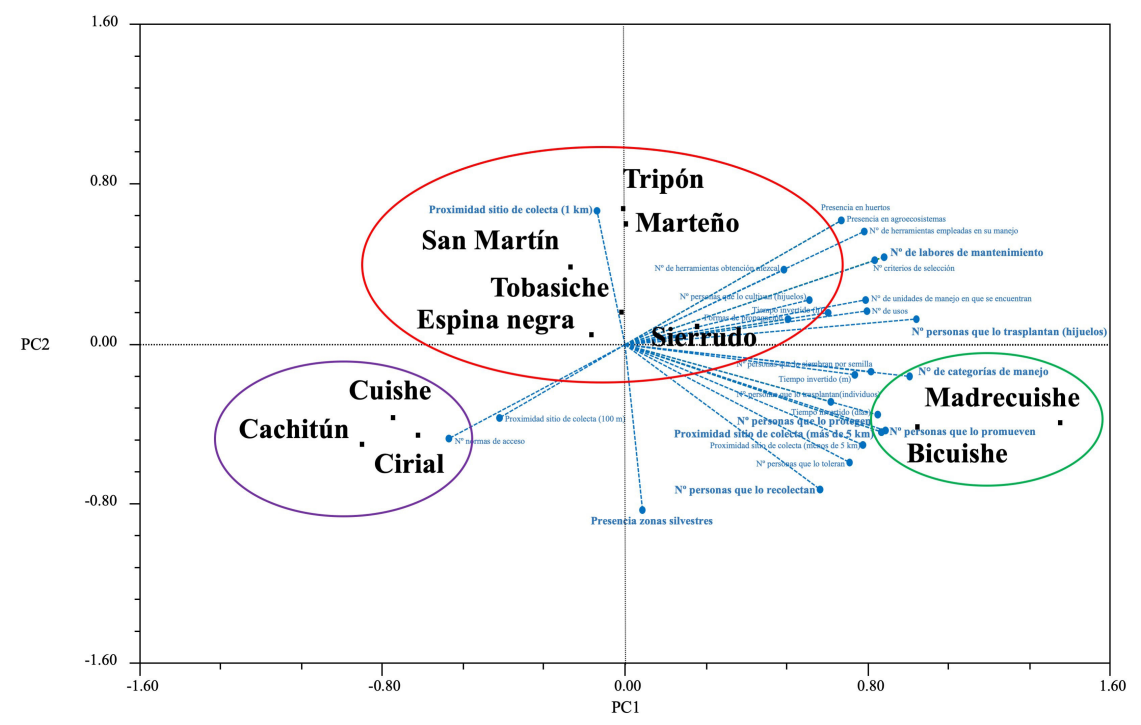

Figura 4. Ordenación de los etnotaxones de A. karwisnkii en función de la intensidad con que son manejados.

reconocimiento cultural de las discontinuidades en la naturaleza (Berlin 1992), ya sea debido a la plasticidad fenotípica de la especie, la heterogeneidad ambiental en que crece, o las fuerzas selectivas humanas que también dan lugar a la diversidad vegetal (Bye 1993). De acuerdo con Beaucage (2012), la gran diversidad y cantidad de nombres con que se conoce a una determinada especie, puede ser un indicio de la importancia cultural de este recurso en su área de distribución natural y tener un sentido práctico, ya que puede estar relacionado con la gran variedad de usos registrados (Hunn 1982).

Los siete usos registrados en $A$. karwinskii contrastan con la gran cantidad que se han reportado para otras especies del género Agave, por ejemplo 14 en A. potatorum (Torres et al. 2013), y 16 en $A$. inaequidens (Torres et al. 2015a; 2016). Destacan los casos de A. salmiana y A. angustifolia con 20 y 16 respectivamente, aunque la primera es una especie domesticada, y la segunda una silvestre con una amplia distribución (Colunga-GarcíaMarín et al. 1996, ColungaGarcíaMarín \& May-Pat 1993).

Las partes útiles documentadas para esta especie concuerdan en general con lo que se ha reportado para muchas especies de agaves (Colunga-GarcíaMarín et al. 2017). No obstante, en A. karwinskii, a pesar de que los tallos y bases foliares se usan para la producción de mezcal, hay etnotaxones que primeramente se seleccionaron como cercos vivos, ya que sus hojas poseen dientes conspicuos, aunque eventualmente también pueden ser aprovechados para hacer mezcal.

La extracción y uso de sus fibras ha caído en desuso, producto de diversos factores, entre estos la sustitución de las fibras naturales por sintéticas. Esto ha sido documentado en diversas especies de agaves que se distribuyen en el estado de Oaxaca (Palma-Cruz 1999) y en otras partes de México (Colunga-GarcíaMarín et al. 2011).

El uso medicinal de las espinas del etnotaxa Cachitún para combatir picaduras de insectos venenosos, es un dato relevante que no había sido documentado en otras especies de agaves. Sin embargo, el uso medicinal es uno de los más amenazados en los agaves. Esto quizás guarde relación con la cada vez más amplia cobertura de los servicios de salud oficiales. Lamentablemente, esta extensión de los servicios médicos también se ha traducido en el abandono de las formas tradicionales de aliviar diversas afecciones, ya que a menudo desde el Estado se ejerce un tipo de coerción social que condiciona la atención médica al abandono de la medicina local $\mathrm{y}$, en muchos casos, se traduce en una desvalorización del uso de las plantas medicinales (ArjonaGarcía 2018).

Se documentó un amplio espectro de formas de manejo: Agave karwinskii está presente en múltiples entornos y forma parte de una matriz que conecta los entornos silvestres con los agroecosistemas (Tabla 1), lo que evidencia que dependiendo del uso, el manejo puede tener distintas intensidades, y eventualmente conducir a procesos de domesticación, tal y como se ha documentado en distintas especies de agave (Colunga-GarcíaMarín et al. 1996, 1997, 2007, 2017, Vargas-Ponce et al. 2007, LeónVázquez et al. 2009, Carrillo-Galván 2011, Delgado-Lemus et al. 2014, Torres et al. 2015a, 2015b, Rivera-Lugo 2018 y en otras plantas de zonas áridas (Blancas et al. 2016).

No obstante, a diferencia de lo que se ha reportado en otras especies de agave, en donde los sistemas y prácticas de manejo se han circunscrito y reducido a entornos antropogénicos; como en los casos de Agave tequilana (Valenzuela-Zapata 1995), A. fourcrodydes (García-Frapolli 

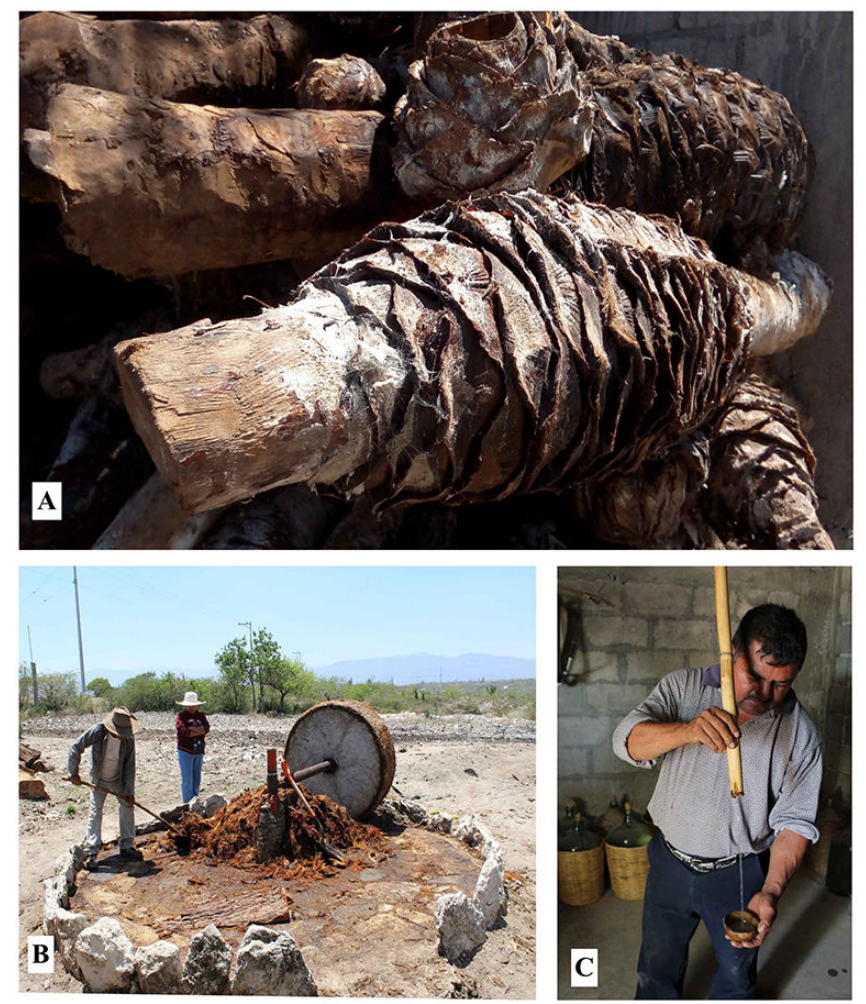

Figura 5. Aprovechamiento de Agave karwinskii para elaboración de mezcal: A) Tallos cocidos ligeramente alargados, característicos del etnotaxón Madrecuishe (Foto: Nancy Vázquez-Pérez); B) Molido y machacado de tallos de A. karwinskii antes de ponerse a fermentar (Foto: Itzel Abad-Fitz); y C) Mezcal de A. karwinskii producido en San Luis Amatlán, Oax. (Foto: Itzel Abad-Fitz).

et al. 2008) y A. salmiana (Martínez-Salvador et al. 2012); en $A$. karwinskii los entornos de manejo y las prácticas son diversas, ya que se presentan formas de manejo tanto in situ como ex situ, este patrón concuerda con lo reportado para otras especies silvestres de agave (Torres et al. 2015a).

Además, cada categoría de manejo puede relacionarse con los etnotaxones que están siendo manipulados, destacando aquellos que son preferidos para la elaboración del mezcal (Tabla 1), los cuales están seriamente amenazados por el auge de esta industria. No obstante, en el pasado A. karwinskii era considerado un maguey de segunda categoría, ya que generalmente se mezclaba con otros agaves a fin de incrementar el rendimiento en la producción de mezcal.

Desafortunadamente la mayoría de los agaves mezcaleros hoy en día enfrentan la disyuntiva de ser aprovechados de manera sostenible conservando la vegetación natural o ser propagados en forma de monocultivos a gran escala a fin de satisfacer la demanda de materia prima (Delgado-Lemus et al. 2014).

De acuerdo con los resultados, las personas han desarrollado criterios de selección en esta especie dependiendo de los usos a los que están destinados. Por ejemplo, para cercos vivos se prefieren Espina negra, Bicuishe, Marteño, Tobasiche y Sierrudo, ya que poseen un porte arborescente y márgenes de las hojas mayormente armados. Esto se ha documentado para A. salmiana var. ferox, ya que es preferida para el diseño de cercos vivos a fin de impedir el paso de ganado a las parcelas de cultivo o simplemente para delimitar los terrenos (Mora-López et al. 2011).

Para la producción de mezcal se prefiere San Martín, Tripón y Madrecuishe por su forma arrosetada y menor tiempo de cosecha, a fin de aprovechar la mayor cantidad de biomasa, porque sus jugos son más dulces y por el rendimiento. Esto ha sido documentado para A. angustifolia var. angustifolia que crece cultivada en el estado de Guerrero (Huerta 2018), en donde el síndrome de domesticación podría estar relacionado con características como el gigantismo (Colunga-GarcíaMarín et al. 1996) y la disminución del tamaño de las espinas y dientes como ocurre en A. mapisaga (Mora-López et al. 2011).

Esta modificación en los criterios de selección no sólo incide en la intensidad de manejo, también está condicionando la presencia de los distintos etnotaxones en los distintos entornos. Ahora se buscan individuos de gran tamaño y de formas arrosetadas ya que son preferidos por su rendimiento. Esto eventualmente puede ir en detrimento de los etnotaxones que se aprovechaban por la fibra (Cuishe y Cirial), o en menor medida de las formas con tallos 
erguidos que se usan como cercos vivos. De esta forma, el mercado parece marcar pautas para la intensificación del manejo, así como de los criterios selectivos (Arellanes et al. 2013, Blancas et al. 2013).

Tabla 2. Índice de intensidad de manejo de los once etnotaxones de A. karwinskii calculado a partir de los registros del primer componente principal (CP1).

\begin{tabular}{lc}
\hline Variedad & Índice de intensidad de manejo \\
\hline Madrecuishe & 1.40 \\
Bicuishe & 1.00 \\
Sierrudo & 0.24 \\
Tobasiche & 0.00 \\
Tripón & -0.20 \\
Marteño & -0.20 \\
Espina Negra & -0.13 \\
San Martín & -0.18 \\
Cirial & -0.70 \\
Cuishe & -0.78 \\
Cachitún & -0.90 \\
\hline
\end{tabular}

Aunque la intensificación en el manejo de los recursos vegetales puede tener algunas barreras y limitaciones, como es la dificultad en su propagación (Blancas et al. 2010), en A. karwinskii no parece ser el caso, ya que se reproduce tanto sexual como asexualmente. Aunque, la forma más favorecida de reproducción es por propagación vegetativa realizada por medio del deshije y trasplante de hijuelos. Esta es una característica de muchas especies de agave, la cual ha sido aprovechada por los humanos para ir fijando características deseables y eventualmente domesticar algunas especies o generar nuevas variedades (Casas et al. 2016). Esta situación entraña otras problemáticas, sobre todo en un contexto de alta demanda de materia prima para la industria del mezcal. Entre estas se puede mencionar la transformación de la diversidad de sistemas de manejo tradicional en plantaciones comerciales que privilegian la propagación clonal, lo que puede llevar a la erosión genética, tal como ocurrió con A. tequilana (EscobarGuzmán 2009, Gil-Vega et al. 2001).

El índice de intensidad de manejo desarrollado en este estudio parece reflejar procesos de domesticación incipiente en A. karwinskii, sobre todo para el etnotaxón Madrecuishe. De acuerdo con Vázquez-Pérez (2015), Bicuishe y Madrecuishe presentan mayores porcentajes de poliploidía $(3 \mathrm{~N})$, en contraste con los etnotaxones no cultivados (Sierrudo, Cirial, Espina negra y Marteño) y que sólo se presentan en la vegetación silvestre, en donde el porcentaje de diploidía $(2 \mathrm{~N})$ es mayor. Se estima que en las angiospermas la poliploidía se presenta entre el 30 y el $60 \%$ de las especies (Soltis et al. 2009). Aunque la poliploidía no es un fenómeno derivado o producto del manejo, éste sí puede aumentar su ocurrencia, sobre todo en aquellas poblaciones sujetas a mayores intensidades de manejo, precisamente porque la poliploidía provee de material genético que puede conferir a las especies una mayor resistencia o mejorar la capacidad para adaptarse a entornos estresantes (Tamayo-Ordóñez et al. 2016). A su vez, las personas pueden echar mano de esta diversidad genética y usarla para su beneficio. Esto parece confirmar lo que en diversas especies de agaves se ha encontrado, que conforme se intensifica el manejo, se favorece la ocurrencia de poblaciones poliploides (Palomino et al. 2008, 2017). Este es el caso de A. fourcroydes Lem., conocida como "Kitam ki" (triploide), A. angustifolia var. letonae (tetraploide),

A. sisalana (pentaploide) y A. angustifolia (variedad "Chelem ki") (hexaploide) (Robert et al. 2008).

También este gradiente de manejo se puede relacionar con las características morfológicas de los distintos etnotaxones de A. karwinskii. Vázquez-Pérez (2015) encontró que el tamaño de la roseta y el tamaño de las semillas de Madrecuishe son más grandes con respecto a las demás variedades. Esto puede estar relacionado con las presiones selectivas que realizan las personas, sobre todo porque este etnotaxón es el más apreciado por la calidad de su destilado. Este mismo estudio encontró que los etnotaxones menos manejados, presentaban mayores niveles de diversidad genética. Sin embargo, también se apreció que los etnotaxones mayormente cultivados como Madrecuishe, presentaban niveles similares de variación genética que los etnotaxones silvestres. Lo anterior puede indicar que la intensificación en el manejo de estos etnotaxones, lejos de disminuir la diversidad genética, la ha mantenido y quizás hasta aumentado. Lo anterior concuerda con lo que se ha observado en poblaciones manejadas y silvestres de A. angustifolia (Vargas-Ponce et al. 2009); A. cupreata (Aguirre-Duguá y Eguiarte 2013); A. potatorum (FélixValdez et al. 2016); y A. inaequidens (Figueredo-Urbina et al. 2017). Esto también se ha encontrado en cactáceas columnares, particularmente del género Stenocereus, en donde se documentaron haplotipos en poblaciones manejadas que no estaban presentes en las poblaciones silvestres (Casas et al. 1997a, 1997b). Particularmente se encontraron colores de pulpa del fruto inexistentes en los ambientes naturales. Lo anterior nos muestra la capacidad de las diversas culturas humanas para mantener e incluso incrementar la diversidad biológica, tal y como lo documentan diversos estudios en Mesoamérica (Casas et al. 2016). 


\section{Conocimiento y manejo tradicional de Agave karwinskii}

Tabla 3. Matriz de cargas mostrando el peso de las variables de intensidad de manejo en los dos primeros componentes principales. Las negritas indican las variables con mayor peso en cada componente principal.

\begin{tabular}{|c|c|c|}
\hline Variable & CP1 & CP2 \\
\hline Formas de propagación & 0.5361 & 0.1215 \\
\hline $\mathrm{N}^{\mathrm{o}}$ de categorías de manejo & 0.9374 & -0.1587 \\
\hline $\mathrm{N}^{\mathrm{o}}$ de usos & 0.7966 & 0.1644 \\
\hline $\mathrm{N}^{\mathrm{o}}$ de personas que lo recolectan & 0.6428 & -0.7297 \\
\hline $\mathrm{N}^{\mathrm{o}}$ de personas que lo toleran & 0.7375 & -0.5918 \\
\hline $\mathrm{N}^{\mathrm{o}}$ de personas que lo promueven & 0.8570 & -0.4298 \\
\hline $\mathrm{N}^{\mathrm{o}}$ de personas que lo protegen & 0.8443 & -0.4429 \\
\hline $\mathrm{N}^{\mathrm{o}}$ de personas que lo trasplantan por hijuelos & 0.9615 & 0.1289 \\
\hline $\mathrm{N}^{\circ}$ de personas que lo trasplantan (individuos completos) & 0.6782 & -0.2882 \\
\hline $\mathrm{N}^{\mathrm{o}}$ de personas que lo cultivan por hijuelos & 0.6057 & 0.2230 \\
\hline $\mathrm{N}^{o}$ de personas que lo siembran por semilla & 0.8106 & -0.1412 \\
\hline $\mathrm{N}^{\mathrm{o}}$ de labores de mantenimiento que recibe & 0.8546 & 0.4332 \\
\hline $\mathrm{N}^{o}$ de herramientas empleadas para su manejo & 0.7868 & 0.5645 \\
\hline $\mathrm{N}^{\mathrm{o}}$ de herramientas usadas para la obtención del mezcal & 0.5233 & 0.3757 \\
\hline $\mathrm{N}^{\mathrm{o}}$ de criterios de selección & 0.8250 & 0.4166 \\
\hline $\mathrm{N}^{\mathrm{o}}$ de medidas para normar el acceso al recurso & -0.5808 & -0.4706 \\
\hline Proximidad de menos $100 \mathrm{~m}$ al sitio de colecta & -0.4141 & -0.3663 \\
\hline Proximidad hasta $1 \mathrm{~km}$ al sitio de colecta & -0.0930 & 0.6637 \\
\hline Proximidad de menos de $5 \mathrm{~km}$ al sitio de colecta & 0.7835 & -0.5084 \\
\hline Proximidad de más de $5 \mathrm{~km}$ al sitio de colecta & 0.8443 & -0.4429 \\
\hline Tiempo invertido (Minutos) & 0.7568 & -0.1500 \\
\hline Tiempo invertido (Horas) & 0.6704 & 0.1543 \\
\hline Tiempo invertido (Días) & 0.8317 & -0.3526 \\
\hline $\mathrm{N}^{o}$ de unidades de manejo en que se encuentra & 0.7930 & 0.2175 \\
\hline Presencia en zonas silvestres & 0.0578 & -0.8345 \\
\hline Presencia en agroecosistemas & 0.7129 & 0.6206 \\
\hline Presencia en huertos & 0.7129 & 0.6206 \\
\hline
\end{tabular}

Agave karwinskii es un recurso regional muy importante en lo ecológico, económico y cultural, aunque la demanda actual lo ha llevado a posicionarse a nivel nacional como una especie intensamente manejada. La diversidad de usos de los distintos etnotaxones ha generado una compleja nomenclatura que indica distintas motivaciones para manejarlas y mantenerlas en diversos entornos, desde los silvestres hasta los transformados por los humanos. Además, refleja una compleja y dinámica historia de desarrollo sociocultural que ha posicionado a determinados productos culturales como claves en lo económico. Entre estos resalta la producción de mezcal con esta especie, el cual es muy apreciado por los consumidores de esta bebida y su demanda aumenta día con día.

La demanda del etnotaxon Madrecuishe en particular se relaciona con las peculiaridades organolépticas de su mezcal, lo que en la actualidad conlleva a la intensificación en el manejo por medio del cultivo, cuyas consecuencias aún están por documentarse. Entre los efectos positivos de la intensificación están el mantener la disponibilidad de agaves, los medios de vida local, arraigando a las personas en sus lugares y creando fuentes de empleo local, además de aprovechar importantes porciones de tierra para incrementar su abundancia. Entre los efectos negativos destaca una 
tendencia creciente al monocultivo, el uso de agroquímicos y la uniformidad de los procesos de extracción, procesamiento y destilado, los cuales van en contra de la diversidad biocultural.

Se advierten fuertes procesos selectivos en Agave karwinskii, los cuales han generado procesos de domesticación incipiente, sobre todo en aquellos etnotaxones que son manejados de forma más intensa. En específico, para la producción del mezcal se seleccionan individuos cuyos tamaños de roseta son más grandes, a fin de obtener mayores rendimientos. Aunque se tienen fuertes indicios de estos procesos, sobre todo con estudios que analizan la diferenciación morfológica, es necesario hacer estudios a nivel genético a fin de corroborar estas hipótesis.

Además, se requiere una evaluación detallada de la disponibilidad espacial de individuos de esta especie en su área de distribución, ya que al parecer la demanda de tallos para la producción de mezcal en la región está excediendo la capacidad de regeneración natural. Esto permitiría delimitar áreas de conservación, reforestación y ordenamiento de la extracción de materia prima con miras a la sustentabilidad de la actividad mezcalera. Al mismo tiempo, es importante indagar el efecto del manejo humano en la producción de flores, frutos y semillas, así como en las diferencias en la tasa de germinación cuando se comparan etnotaxa sujetos a distintas intensidades de manejo. Lo anterior constituye una estrategia de investigación, con miras a proponer en un futuro estrategias de manejo sostenible para esta especie.

La documentación de los conocimientos y formas de manejo tradicional en $A$. karwinskii resultan cruciales, a fin de mantener y conservar la biodiversidad local, pero también los medios de vida de las comunidades locales.

\section{Agradecimientos}

A las comunidades en donde se realizó el presente estudio, por la confianza para compartir sus conocimientos. Al Doctorado en Ciencias Naturales de la Universidad Autónoma del Estado de Morelos. Al Consejo Nacional de Ciencia y Tecnología por la beca para poder llevar a cabo esta investigación. A la Red Temática Productos Forestales No Maderables: aportes desde la etnobiología para su aprovechamiento sostenible (Proyectos CONACYT 271837, 280901), por el financiamiento para realizar el trabajo de campo.

\section{Literatura citada}

Arellanes Y, Casas A, Arellanes A, Vega E, Blancas J, Vallejo M, Torres I, Rangel-Landa S, Moreno A, Solís L, Pérez-Negrón E. 2013. Influence of traditional markets on plant management in the Tehuacan Valley. Journal of
Ethnobiology and Ethnomedicine 9. DOI: http://doi.org/ 10.1186/1746-4269-9-38.

Arjona-García C. 2018. Urbanización como proceso de cambio en el uso de plantas medicinales en tres comunidades de la Sierra de Huautla en el estado de Morelos. MSc. Thesis, Universidad Autónoma del Estado de Morelos.

Arnaiz-Schmitz C, Herrero-Jáuregui C, Schmitz MF. 2018. Losing a heritage hedgerow landscape. Biocultural diversity conservation in a changing social-ecological Mediterranean system. Science of the Total Environment 637-638: 374-384. DOI: https://doi.org/10.1016/j.scit otenv.2018.04.413

Beaucage P. 2012. Cuerpo, cosmos y medio ambiente entre los nahuas de la Sierra Norte de Puebla. México, DF: Plaza y Valdés. ISBN: 978-607-402-533-0

Beltrán-Rodríguez L, Manzo-Ramos F, MaldonadoAlmanza B, Martínez-Ballesté A, Blancas J. 2017. Wild medicinal species traded in the Balsas Basin, Mexico: risk analysis and recommendations for their conservation. Journal of Ethnobiology 37: 743-764. DOI: http://doi.org/10.2993/0278-0771-37.4.743

Berkes F. 1999. Sacred Ecology. Philadelphia: Taylor \& Francis. ISBN: 1-56032-695-6

Berkes F, Folke C. 1998. Linking Social and Ecological Systems. Management practices and social mechanisms for building resilience. Cambridge: Cambridge University Press. ISBN: 978-0521785624

Berlin B. 1992. Ethnobiological Classification: Principles of Categorization of Plants and Animals in Traditional Societies. New Jersey: Princeton University Press. ISBN: 978-1-4008-6259-7

Bernard R. 2011. Research Methods in Anthropology: Qualitative and Quantitative Approaches. Maryland: Altamira Press. ISBN: 978-0-7591-1241-4

Blancas J, Casas A, Moreno-Calles AI, Caballero J. 2016. Cultural motives of plants management and domestication. In: Lira $\mathrm{R}$, Casas A, Blancas J. Ethnobotany of Mexico. Interactions of People and Plants in Mesoamerica. New York: Springer, pp. 233-256. ISBN: 978-1-4614-6668-0

Blancas J, Casas A, Pérez-Salicrup D, Caballero J, Vega E. 2013. Ecological and socio-cultural factors influencing plant management in Náhuatl communities of the Tehuacán Valley, Mexico. Journal of Ethnobiology and Ethnomedicine 9. DOI: http://doi.org/10.1186/1746-4269 $\underline{-9-39}$

Blancas J, Casas A, Rangel-Landa S, Moreno-Calles A, Torres I, Pérez-Negrón E, Solís L, Delgado-Lemus A, Parra F, Arellanes Y, Caballero J, Cortés L, Lira R, Dávila P. 2010. Plant Management in the TehuacanCuicatlan Valley, Mexico. Economic Botany 64: 
287-302. DOI: http://doi.org/10.1007/s12231-010-9133 $\underline{-0}$

Boege E. 2008. El patrimonio biocultural de los pueblos indigenas de México. Hacia la conservación in situ de la biodiversidad y agrodiversidad en los territorios indigenas. México DF: Instituto Nacional de Antropología e Historia - Comisión Nacional para el Desarrollo de los Pueblos Indígenas. ISBN: 978-968-03-0385-4

Brush SB. 1993. Indigenous knowledge of biological resources and intellectual property rights: the role of anthropology. American Anthropologist 95: 653-671. DOI: http://doi.org/10.1525/aa.1993.95.3.02a00060

Bye RA. 1993. The role of humans in the diversification of plants in Mexico. In: Ramamoorthy $\mathrm{P}$, Bye R, Lot A. Biological Diversity of Mexico: Origins and distribution. New York: Oxford University Press Inc. pp. 707-731. ISBN: 978-0195066746

Carrillo-Galván MG. 2011. Domesticación de agaves productores de fibra en el centro-occidente de México: una aproximación etnobotánica y morfológica. MSc. Thesis, Centro de Investigaciones Científicas de Yucatán AC.

Casas A, Caballero J, Mapes C, Zárate S. 1997a. Manejo de la vegetación, domesticación de plantas y origen de la agricultura en Mesoamérica. Botanical Sciences 61: 3147. DOI: http://doi.org/10.17129/botsci.1537

Casas A, Pickersgill B, Caballero J, Valiente-Banuet A. 1997b. Ethnobotany and domestication in Xoconochtli Stenocereus stellatus (Cactaceae), in the Tehuacán Valley and La Mixteca Baja, México. Economic Botany 51: 279-292. DOI: https://doi.org/10.1007/BF02862097

Casas A, Torres-Guevara J, Parra F. eds. 2016. Domesticación en el continente americano. Manejo de biodiversidad y evolución dirigida por las culturas del Nuevo Mundo. Volumen 1. Lima: Universidad Nacional Autónoma de México - Universidad Agraria La Molina. ISBN: 978-612-4147-59-3

Colunga-GarcíaMarín P, Carrillo-Galván G, ZizumboVillarreal D, Vargas-Ponce O. 2011. Agaves ixtleros del sur de Jalisco. Mérida: Centro de Investigación Científica de Yucatán, AC. ISBN: 978-607-7823-09-4

Colunga-GarcíaMarín P, Coello-Coello J, Espejo-Peniche L, Fuente-Moreno L. 1993. Agave studies in Yucatan, Mexico. II. Nutritional value of the inflorescence peduncle and incipient domestication. Economic Botany 47: 328-334. DOI: https://doi.org/10.1007/BF02862302

Colunga-GarcíaMarín P, Estrada-Loera E, May-Pat F. 1996. Patterns of morphological variation, diversity, and domestication of wild and cultivated populations of Agave in Yucatan, Mexico. American Journal of Botany 83: 1069-1082. DOI: http://doi.org/10.2307/2445997
Colunga-GarcíaMarín P, May-Pat F. 1993. Agave studies in Yucatan, Mexico. I. Past and present germplasm diversity and uses. Economic Botany 47: 312-327. DOI: http://doi.org/10.1007/BF02862301

Colunga-GarcíaMarín P, May-Pat F. 1997. Morphological variation of henequen (Agave fourcroydes, Agavaceae) germoplasm and its wild ancestor (A. angustifolia) under uniform growth conditions: diversity and domestication. American Journal of Botany 84:1449-1465. DOI: https:// doi.org/10.2307/2446608

Colunga-GarcíaMarín P, Torres-García I, Casas A, Figueredo-Urbina CJ, Rangel-Landa S, Delgado-Lemus A, Vargas O, Cabrera-Toledo D, Aguirre-Dugua, Eguiarte L, Zizumbo-Villarreal D, Carrillo-Galván G. 2017. Los agaves y las prácticas mesoamericanas de aprovechamiento, manejo y domesticación. In: Casas A, Torres-Guevara J, Parra F. eds. Domesticación en el Continente Americano. Manejo de Biodiversidad y Evolución Dirigida por las Culturas del Nuevo Mundo. Volumen 2. pp. 273-308. Morelia: Universidad Nacional Autónoma de México-Universidad Nacional Agraria de La Molina,

Colunga-GarcíaMarín P, Zizumbo-Villarreal D. 2006. Tequila and other Agave spirits from west-central Mexico: current germplasm diversity, conservation and origin. Biodiversity and Conservation 16: 1653-1667. DOI: http://doi.org/10.1007/s10531-006-9031-z

Colunga-GarcíaMarín P, Zizumbo-Villareal D. 2007. El tequila y otros mezcales del centro-occidente de México: domesticación, diversidad y conservación de germoplasma. In: Colunga-GarcíaMarín $\mathrm{P}$, Larqué $\mathrm{A}$, Eguiarte L, Zizumbo-Villareal D. eds. En lo Ancestral hay Futuro: del Tequila, los Mezcales y Otros Agaves. Mérida: Centro de Iinvestigación Científica de YucatánConsejo Nacional de Ciencia y Tecnología-Comisión Nacional para el Conocimiento y Uso de la Biodiversidad-Instituto Nacional de Ecología, pp. 113-131.

CONEVAL [Consejo Nacional de Evaluacion de la Politica de Desarrollo Social]. 2017. Medición de pobreza. Indicadores de carencia social. https://www.coneval. org.mx/Medicion/Paginas/Medición/Indicadores-decarencia-social.aspx (accessed July 18, 2019).

Delgado-Lemus A, Torres I, Blancas J, Casas A. 2014. Vulnerability and risk management of Agave species in the Tehuacán Valley, México. Journal of Ethnobiology and Ethnomedicine 10. DOI: http://doi.org/10.1186/ 1746-4269-10-53

Drew JA, Henne AP. 2006. Conservation biology and traditional ecological knowledge: Integrating academic disciplines for better conservation practice. Ecology and 
Society 11. DOI: http://doi.org/10.5751/ES-01959$\underline{110234}$

Escobar-Guzmán RE. 2009. Estudio de la biología reproductiva y análisis molecular de la reproducción sexual y asexual de Agave tequilana Weber var. azul. $\mathrm{PhD}$. Thesis. Instituto Politécnico Nacional.

Félix-Valdez LI, Vargas-Ponce O, Cabrera-Toledo D, Casas A, Cibrian-Jaramillo A, Cruz-Larios L. 2016. Effects of traditional management for mescal production on the diversity and genetic structure of Agave potatorum (Asparagaceae) in central Mexico. Genetic Resources and Crop Evolution 63: 1255-1271. DOI: http://doi.org/ 10.1007/s10722-015-0315-6

Figueredo CJ, Casas A, Colunga-GarcíaMarín P, Nassar JM， González-Rodríguez A. 2014. Morphological variation, management and domestication of 'maguey alto' (Agave inaequidens) and "maguey manso" (A. hookeri) in Michoacán, México. Journal of Ethnobiology and Ethnomedicine 10. DOI: http://doi.org/10.1186/ 1746-4269-10-66

Figueredo-Urbina CJ, Casas A, Torres-García I. 2017. Morphological and genetic divergence between Agave inaequidens, A. cupreata and the domesticated $A$. hookeri. Analysis of their evolutionary relationships. PloS One 12. DOI: http://doi.org/10.1371/journal. pone. 0187260

García-Frapolli E, Toledo VM, Martinez-Alier J. 2008. Adaptations of a Yucatec Maya Multiple-Use Ecological Management Strategy to Ecotourism. Ecology and Society 13. DOI: https://doi.org/10.5751/ES-02627130231

García-Mendoza A, Galván VR. 1995. Richness of the Agavaceae and Nolinaceae in Mexico. Botanical Sciences 56: 7-24. DOI: http://doi.org/10.17129/botsci. 1461

García-Mendoza AJ, Franco-Martínez IS, SandovalGutiérrez D. 2019. Cuatro especies nuevas de Agave (Asparagaceae, Agavoideae) del sur de México. Acta Botanica Mexicana 126: 1-18. DOI: https://doi.org/ $\underline{10.21829 / \mathrm{abm} 126.2019 .1461}$

Gil-Vega K, González-Chavira M, Martínez-de la Vega O, Simpson J, Vandemark G. 2011. Analysis of genetic diversity in Agave tequilana var. azul using RAPD markers. Euphytica 119: 335-341. DOI: https://doi.org/ 10.1023/A:1017553107303

Gobierno del Estado de Oaxaca. 2015. Planes de Desarrollo Municipal. Secretaría de Finanzas. < https:// www.finanzasoaxaca.gob.mx/pdf/inversion_publica/ pmds/11 13/235.pdf > (accessed July 18, 2019).

González-Insuasti MS, Caballero J. 2007. Managing Plant Resources: How Intensive Can it be? Human Ecology 35: 303-314. DOI: http://doi.org/10.1007/s10745-006-90 $\underline{63-8}$
Gunderson LH, Holling CS. (Ed.). 2002. Washington DC. Island Press. ISBN: 1559638575

Hersch-Martínez P. 1995. Commercialization of wild medicinal plants from Southwest Puebla, Mexico. Economic Botany 49: 197-206. DOI: http://doi.org/ 10.1007/BF02862925

Hersch-Martínez P. 1997. Medicinal plants and regional traders in Mexico: Physiographic differences and conservational challenge. Economic Botany 51: 107-120. DOI: http://doi.org/10.1007/BF02893100

Hildebrand EA. 2003. Motives and opportunities for domestication: An ethnoarchaeological study in southwest Ethiopia. Journal of Anthropological Archaeology, 22: 358-375. DOI: http://doi.org/10.1016/ $\underline{\mathrm{S} 0278-4165(03) 00031-\mathrm{X}}$

Huerta J. 2018. Variación morfológica y áreas potenciales de poblaciones del complejo Agave angustifolia en el estado de Guerrero. MSc. Thesis, Universidad Autónoma del Estado de Guerrero.

Hunn E. 1982. The utilitarian factor in folk biological classification. American Anthropologist 84: 830-847. DOI: http://doi.org/10.1525/aa.1982.84.4.02a00070

INPI [Instituto Nacional de los Pueblos Indígenas]. 2017. Indicadores Socioeconómicos de los Pueblos Indígenas de México, 2015. https://www.gob.mx/inpi/articulos/ indicadores-socioeconomicos-de-los-pueblos-indigenasde-mexico-2015-116128 (accessed July 18, 2019).

Kates RW, Clark WC, Corell R, Hall JM, Jaeger CC, Lowe I, McCarthy J, Schellnhuber H, Bolin B, Dickson N, Faucheux S, Gallopin G, Grübler A, Huntley B, Jäger J, Jodha N, Kasperson R, Mabogunje A, Matson P, Mooney H, Moore III B, O'Riordan T, Svedin U. 2001. Environment and development: Sustainability science. Science 292: 641-642. DOI: http://doi.org/10.1126/ science. 1059386

Lima-da Silva T, de Almeida-Campos J, Chaves-Alves Â, Albuquerque U. 2019. Market integration does not affect traditional ecological knowledge but contributes additional pressure on plant resources. Acta Botanica Brasilica 33: 1-9. DOI: http://doi.org/10.1590/0102-3306 $\underline{2018 \mathrm{abb} 0310}$

León-Vázquez NI, Campos-Ángeles GV, Enríquez-del Valle JR, Velasco-Velasco VA, Marini-Zúñiga F, RodríguezOrtiz G. 2013. Diversidad de especies de agave en San Miguel Tilquiapam, Ocotlán, Oaxaca. Revista Mexicana De Ciencias Agrícolas 6: 1185-1195.

Martin GJ. 2004. Ethnobotany: a methods manual. New York: Taylor \& Francis. ISBN: 978-1-84407-084-8

Martínez-Ballesté A, Martorell C, Caballero J. 2006. Cultural or ecological sustainability? The effect of cultural change on Sabal palm management among the lowland Maya of Mexico. Ecology and Society 11. DOI: http://doi.org/10.5751/ES-01803-110227 
Martínez-Salvador M, Mata-González R, Morales C, Valdez-Cepeda R. 2012. Agave salmiana plant communities in central Mexico as affected by commercial use. Environmental Management, 49: 55-63. DOI: http://doi.org/10.1007/s00267-011-9759-4

Menzies CR. ed. 2006. Traditional ecological knowledge and natural resource management. Lincoln: University of Nebraska Press. ISBN: 978-0-8032-8319-0

Mora-López JL, Reyes-Agüero JA, Flores-Flores JL, PeñaValdivia CB, Aguirre-Rivera JR. 2011. Variación morfológica y humanización de la sección Salmianae del género Agave. Agrociencia 45: 465-477.

Moreno-Calles AI, Casas A, Toledo VM, Vallejo-Ramos M. (coords.). 2016. Etnoagroforestería en México. Morelia: Universidad Nacional Autónoma de México. ISBN: 978-607-02-8164-8

Palma-Cruz FJ. 1999. Fibras duras de Ixtle (Agave): especies productoras y formas de utilización en el estado de Oaxaca. BSc. Thesis, Universidad Nacional Autónoma de México.

Palomino G, Martínez J, Méndez I. 2008. Karyotype studies in cultivars of Agave tequilana Weber. Caryologia 61: 144-153. DOI: http://doi.org/10.1080/00087114.2008. $\underline{10589622}$

Palomino G, Martínez J, Romero P, Barba-González R, Rodríguez-Garay B. 2017. Nuclear genome size and karyotype analysis of Agave angustifolia Haw. “Cimarron” and 'Lineño' (Asparagales, Asparagaceae). Caryologia 70: 93-101. DOI: http://doi.org/10.1080/ $\underline{00087114.2017 .1279931}$

Rangel-Landa S, Casas A, García-Frapolli E, Lira R. 2017. Sociocultural and ecological factors influencing management of edible and non-edible plants: the case of Ixcatlán, Mexico. Journal of Ethnobiology and Ethnomedicine 13. DOI: http://doi.org/10.1186/s13002 -017-0185-4

Rivera-Lugo M, García-Mendoza A, Simpson J, Solano E, Gil-Vega K. 2018. Taxonomic implications of the morphological and genetic variation of cultivated and domesticated populations of the Agave angustifolia complex (Agavoideae, Asparagaceae) in Oaxaca, Mexico. Plant Systematics and Evolution 304: 969-979. DOI: http://doi.org/10.1007/s00606-018-1525-0

Robert ML, Lim Y, Hanson L, Sánchez-Teyer F, Bennett M, Leitch A, Leitch I. 2008. Wild and agronomically important Agave species (Asparagaceae) show proportional increases in chromosome number, genome size, and genetic markers with increasing ploidy. Botanical Journal of the Linnean Society 158: 215-222. DOI: $\underline{\text { https://doi.org/10.1111/j.1095-8339.2008.00831.x }}$

Rohlf FJ. 2000. NTSYS-pc: Numerical Taxonomy and Multivariate Analysis System, V.2.2. Setauket, New York: Exeter Software.
Saynes-Vásquez A, Caballero J, Meave JA, Chiang F. 2013. Cultural change and loss of ethnoecological knowledge among the Isthmus Zapotecs of Mexico. Journal of Ethnobiology and Ethnomedicine 9. DOI: http://doi.org/ $\underline{10.1186 / 1746-4269-9-40}$

Saynes-Vásquez A, Vergara-Silva F, Caballero J. 2016. An interdisciplinary perspective on the loss of Traditional Ecological Knowledge (TEK) in the Tehuantepec Isthmus, Oaxaca. In: Lira R, Casas A, Blancas J. Ethnobotany of Mexico. Interactions of People and Plants in Mesoamerica. pp. 457-473. New York: Springer, DOI: http://doi.org/10.1007/978-1-4614-6669 $-7 \quad 19$

SEDESOL [Secretaría de Desarrollo Social]. 2010. Unidad de microregiones. Cédulas de Información Municipal (SCIM). Sistema de Apoyo para la Planeación del PDZP. http://www.microrregiones.gob.mx/zap/default.aspx?ent $\underline{\mathrm{ra}=\text { nacion }}$ (accessed July 18, 2019).

Shackleton CM, Pandey AK, Ticktin T. 2015. Ecological sustainability for non-timber forest products. New York: Routledge. ISBN: 978-0-415-72859-1

Soltis DE, Albert VA, Leebens-Mack J, Bell CD, Paterson AH, Zheng C, Sankoff D, dePamphilis C, Wall K, Soltis P. 2009. Polyploidy and angiosperm diversification. American Journal of Botany 96: 336-348. DOI: http:// doi.org/10.3732/ajb.0800079

Tamayo-Ordóñez MC, Rodriguez-Zapata LC, NarváezZapata JA, Tamayo-Ordóñez YJ, Ayil-Gutiérrez BA, Barredo-Pool F, Sánchez-Teyer LF. 2016. Morphological features of different polyploids for adaptation and molecular characterization of CC-NBS-LRR and LEA gene families in Agave L. Journal of Plant Physiology 195: 80-94. DOI: http://doi.org/10.1016/j.jplph.2016.03. $\underline{009}$

Tang R, Gavin MC. 2016. A classification of threats to traditional ecological knowledge and conservation responses. Conservation and Society 14: 57-70. DOI: http://doi.org/10.4103/0972-4923.182799

Toledo VM, Barrera-Bassols N. 1984. Ecología y desarrollo rural en Pátzcuaro: un modelo para el análisis interdisciplinario de comunidades campesinas. México DF: Universidad Nacional Autónoma de México.

Toledo VM, Barrera-Bassols N. 2008. La memoria biocultural. La importancia ecológica de las sabidurías tradicionales. Barcelona: Junta de Andalucía/Icaria Editorial. ISBN: 978-84-9888-001-4

Torres I, Blancas J, León A, Casas A. 2015a. TEK, local perceptions of risk, and diversity of management practices of Agave inaequidens in Michoacán, Mexico. Journal of Ethnobiology and Ethnomedicine 11. DOI: http://doi.org/10.1186/s13002-015-0043-1 
Torres I, Casas A, Delgado-Lemus A, Rangel-Landa S. 2013. Aprovechamiento, demografía y establecimiento de Agave potatorum en el Valle de Tehuacán, México: Aportes ecológicos y etnobiológicos para su manejo sustentable. Zonas Áridas 15: 92-109. DOI: http:// dx.doi.org/10.21704/za.v15i1.110

Torres I, Casas A, Delgado-Lemus A, Rangel-Landa S. 2016. Aprovechamiento, demografía y establecimiento de Agave potatorum en el Valle de Tehuacán, México: Aportes ecológicos y etnobiológicos para su manejo sustentable. Zonas Áridas 15: 92-109. DOI: http:// doi.org/10.21704/za.v15i1.110

Torres I, Casas A, Vega E, Martínez-Ramos M, DelgadoLemus A. 2015b. Population Dynamics and Sustainable Management of Mescal Agaves in Central Mexico: Agave potatorum in the Tehuacán-Cuicatlán Valley. Economic Botany 69: 26-41. DOI: https://doi.org/ 10.1007/s12231-014-9295-2

Torres-García I, Rendón-Sandoval FJ, Blancas J, Casas A, Moreno-Calles AI. 2019. The genus Agave in agroforestry systems of Mexico. Botanical Sciences, 97: 263-290. DOI: https://doi.org/10.17129/botsci.2202

Valenzuela-Zapata AG. 1995. La agroindustria del agave tequilero Agave tequilana Weber. Botanical Sciences 55: 15-11. DOI: http://doi.org/10.17129/botsci.1473

Editor de sección: Andrea Martínez Ballesté

Contribución de los autores: NVP diseñó la entrevista, obtuvo los datos en campo y revisó el manuscrito. JB analizó los datos y escribió el manuscrito. ITG analizó los datos y revisó el manuscrito. AGM analizó los datos y revisó el manuscrito. AC escribió y revisó el manuscrito. AIMC revisó el manuscrito. BMA revisó el manuscrito. BRA revisó el manuscrito.
Vargas-Ponce O, Zizumbo-Villarreal D, ColungaGarcíaMarin P. 2007. In situ diversity and maintenance of traditional Agave landraces used in spirits production in West-Central Mexico. Economic Botany 61: 362-375. DOI: https://doi.org/10.1663/0013-0001(2007)61[362:IS DAMO]2.0.CO;2

Vargas-Ponce O, Zizumbo-Villareal D, Martínez-Castillo J, Coello-Coello J, Colunga-GarcíaMarín $\quad$ P. 2009. Diversity and structure of landraces of Agave grown for spirits under traditional agriculture: A comparison with wild populations of A. angustifolia (Agavaceae) and commercial plantations of A. tequilana. American Journal of Botany 96: 448-457. DOI: https://doi.org/ 10.3732/ajb.0800176

Vázquez-Pérez N. 2015. Variación morfológica y genética de Agave karwinskii (Agavaceae) en los estados de Oaxaca y Puebla. 81 pp. MSc. Thesis. Universidad Nacional Autónoma de México.

Zizumbo-Villarreal D, Vargas-Ponce O, Rosales-Adame JJ, Colunga-GarcíaMarín P. 2012. Sustainability of the traditional management of Agave genetic resources in the elaboration of mezcal and tequila spirits in western Mexico. Genetic Resources and Crop Evolution 60: 33-47. DOI: http://doi.org/10.1007/s10722-012-9812-z 
Apéndice 1. Información ambiental de las localidades estudiadas.

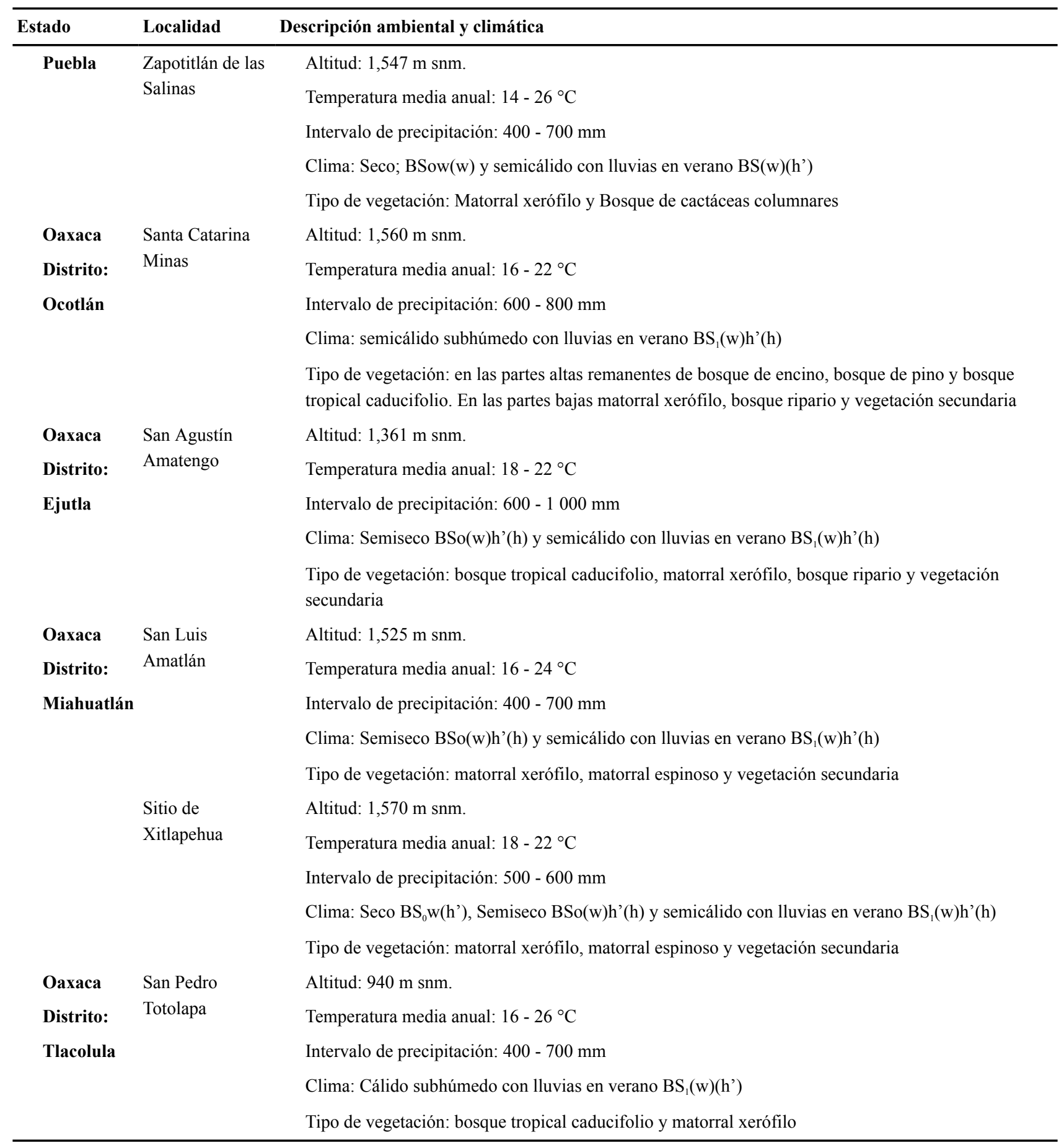


Vázquez-Pérez et al. / Botanical Sciences 98(2): 328-347. 2020

Apéndice 2. Información socioeconómica de las localidades estudiadas (E-SE = entrevista semiestructurada; $\mathrm{E}-\mathrm{A}=$ entrevista abierta).

\begin{tabular}{|c|c|c|c|c|c|}
\hline $\begin{array}{l}\text { Localidad / Grado } \\
\text { de marginación* }\end{array}$ & $\begin{array}{l}\mathrm{N}^{0} \text { total de } \\
\text { habitantes / } \\
\text { mujeres, } \\
\text { hombres** }\end{array}$ & $\begin{array}{l}\text { Grupos } \\
\text { Étnicos / } \\
\text { porcentaje } \\
\text { hablantes de } \\
\text { lengua } \\
\text { indígena*** }\end{array}$ & $\begin{array}{l}\text { Actividades económicas } \\
* * * *\end{array}$ & $\begin{array}{l}\text { Tipo de } \\
\text { tenencia de la } \\
\text { tierra } * * * *\end{array}$ & $\begin{array}{l}N^{0} \text { entrevistados / tipo de } \\
\text { entrevista }\end{array}$ \\
\hline $\begin{array}{l}\text { Zapotitlán de las } \\
\text { Salinas / Alto }\end{array}$ & $\begin{array}{l}2,700 / \\
1433 \\
1204\end{array}$ & $\begin{array}{l}\text { Popoloca y } \\
\text { Mestizo / 22\% }\end{array}$ & $\begin{array}{l}\text { Agricultura de temporal y } \\
\text { ecoturismo }\end{array}$ & $\begin{array}{l}\text { Ejidal y } \\
\text { comunal }\end{array}$ & $\begin{array}{l}5 \text { hombres } \\
2 \text { mujeres } \\
\text { (E-SE) } \\
2 \text { hombres } \\
2 \text { mujeres } \\
(\mathrm{E}-\mathrm{A})\end{array}$ \\
\hline $\begin{array}{l}\text { Santa Catarina } \\
\text { Minas / Medio }\end{array}$ & $\begin{array}{l}1,816 / \\
927,889\end{array}$ & Zapoteco / 2.3\% & $\begin{array}{l}\text { Agricultura de temporal, } \\
\text { producción de mezcal y } \\
\text { servicios }\end{array}$ & $\begin{array}{l}\text { Comunal y } \\
\text { pequeña } \\
\text { propiedad }\end{array}$ & $\begin{array}{l}3 \text { hombres } \\
5 \text { mujeres } \\
\text { (E-SE) } \\
3 \text { hombres } \\
4 \text { mujeres } \\
\text { (E-A) }\end{array}$ \\
\hline $\begin{array}{l}\text { San Agustín } \\
\text { Amatengo / Alto }\end{array}$ & $\begin{array}{l}1,455 / \\
745,614\end{array}$ & Zapoteco / 0.5\% & $\begin{array}{l}\text { Agricultura de temporal, } \\
\text { producción de mezcal y } \\
\text { artesanías de palma }\end{array}$ & $\begin{array}{l}\text { Ejidal y pequeña } \\
\text { propiedad }\end{array}$ & $\begin{array}{l}2 \text { hombres } \\
3 \text { mujeres } \\
\text { (E-SE) } \\
3 \text { hombres } \\
3 \text { mujeres } \\
\text { (E-A) }\end{array}$ \\
\hline $\begin{array}{l}\text { San Luis Amatlán / } \\
\text { Alto }\end{array}$ & $\begin{array}{l}3,624 / \\
1967 \\
1657\end{array}$ & Zapoteco / 1.5\% & $\begin{array}{l}\text { Agricultura de temporal, } \\
\text { producción de mezcal y } \\
\text { extracción de leña }\end{array}$ & $\begin{array}{l}\text { Ejidal, comunal } \\
\text { y pequeña } \\
\text { propiedad }\end{array}$ & $\begin{array}{l}8 \text { hombres } \\
8 \text { mujeres } \\
\text { (E-SE) } \\
12 \text { hombres } \\
12 \text { mujeres } \\
\text { (E-A) }\end{array}$ \\
\hline $\begin{array}{l}\text { Sitio de } \\
\text { Xitlapehua / Alto }\end{array}$ & $\begin{array}{l}705 / \\
366,339\end{array}$ & Zapoteco / 5.8\% & $\begin{array}{l}\text { Agricultura de temporal, } \\
\text { producción de mezcal y } \\
\text { ganadería extensiva }\end{array}$ & $\begin{array}{l}\text { Pequeña } \\
\text { propiedad }\end{array}$ & $\begin{array}{l}1 \text { mujer } \\
(\mathrm{E}-\mathrm{A})\end{array}$ \\
\hline $\begin{array}{l}\text { San Pedro } \\
\text { Totolapa / Medio }\end{array}$ & $\begin{array}{l}2,603 / \\
1376 \\
1227\end{array}$ & Zapoteco / 12.1\% & $\begin{array}{l}\text { Agricultura de temporal, } \\
\text { producción de mezcal y } \\
\text { minería en pequeña escala } \\
\text { (piedra, arena y grava) }\end{array}$ & $\begin{array}{l}\text { Ejidal, comunal } \\
\text { y pequeña } \\
\text { propiedad }\end{array}$ & $\begin{array}{l}3 \text { hombres } \\
5 \text { mujeres } \\
\text { (E-SE) } \\
3 \text { hombres } \\
5 \text { mujeres } \\
\text { (E-A) }\end{array}$ \\
\hline
\end{tabular}




\section{Conocimiento y manejo tradicional de Agave karwinskii}

Apéndice 3. Descripción de las variables de intensidad de manejo de los etnotaxones de A. karwinskii.

\begin{tabular}{l} 
Variable \\
\hline Formas de propagación \\
$\mathrm{N}^{\circ}$ de categorías de manejo \\
$\mathrm{N}^{\circ}$ de usos \\
$\mathrm{N}^{\circ}$ personas que lo recolectan \\
$\mathrm{N}^{\circ}$ personas que lo toleran \\
$\mathrm{N}^{\circ}$ personas que lo promueven \\
$\mathrm{N}^{\circ}$ personas que lo protegen \\
$\mathrm{N}^{\circ}$ personas que lo trasplantan por hijuelos \\
$\mathrm{N}^{\circ}$ personas que lo trasplantan individuos \\
$\mathrm{Completos}^{\circ}$ \\
$\mathrm{N}^{\circ}$ personas que lo cultivan por hijuelos \\
$\mathrm{N}^{\circ}$ personas que lo siembran por semilla \\
$\mathrm{N}^{\circ}$ de labores de mantenimiento
\end{tabular}

$\mathrm{N}^{\mathrm{o}}$ de herramientas

$\mathrm{N}^{\mathrm{o}}$ de herramientas obtención mezcal

$\mathrm{N}^{\mathrm{o}}$ de criterios de selección

$\mathrm{N}^{\mathrm{o}}$ de medidas para normar el acceso al recurso

Proximidad de menos $100 \mathrm{~m}$ al sitio de colecta

Proximidad hasta $1 \mathrm{~km}$ al sitio de colecta

Proximidad de menos de $5 \mathrm{~km}$ al sitio de colecta

Proximidad de más de $5 \mathrm{~km}$ al sitio de colecta Número de entrevistados que refirió recorrer esta distancia a los sitios de colecta y manejo.

Tiempo invertido (Minutos)

Tiempo invertido (Horas)

Tiempo invertido (Días)

$\mathrm{N}^{\circ}$ de unidades de manejo en que se encuentra

Presencia en zonas silvestres

Presencia en agroecosistemas

Presencia en huertos

\section{Descripción/Ponderación}

Tipo de propagación practicada / asexual (1); sexual (2); ambas (3)

Número de categorías de manejo identificadas, se consideran tanto in situ como ex situ. Número total de usos.

Número de personas que realizan esta práctica sobre el universo de 43 entrevistados. Número de personas que realizan esta práctica sobre el universo de 43 entrevistados. Número de personas que realizan esta práctica sobre el universo de 43 entrevistados. Número de personas que realizan esta práctica sobre el universo de 43 entrevistados. Número de personas que realizan esta práctica sobre el universo de 43 entrevistados. Número de personas que realizan esta práctica sobre el universo de 43 entrevistados.

Número de personas que realizan esta práctica sobre el universo de 43 entrevistados. Número de personas que realizan esta práctica sobre el universo de 43 entrevistados. Número de labores, como deshierbe, deshije, arrime de tierra, abonado, eliminación de plagas, construcción de surcos para absorción de agua, ablandamiento del suelo, remoción de hojas secas y encalado.

Número de herramientas usadas en las labores de mantenimiento.

Número de herramientas usadas específicamente para la obtención del mezcal.

Selección por color, aspecto, vigor, grosor del tallo, hojas fibrosas, hojas con mayor presencia de espinas, floración prematura, etc.

Normatividad interna para acceder al recurso / no existe (0); existe, pero no se aplica (1); existe y se aplica (2)

Número de entrevistados que refirió recorrer esta distancia a los sitios de colecta y manejo.

Número de entrevistados que refirió recorrer esta distancia a los sitios de colecta y manejo.

Número de entrevistados que refirió recorrer esta distancia a los sitios de colecta y manejo. Número de entrevistados que indicó invertir este tiempo en las labores de mantenimiento y obtención de la materia prima.

Número de entrevistados que indicó invertir este tiempo en las labores de mantenimiento y obtención de la materia prima.

Número de entrevistados que indicó invertir este tiempo en las labores de mantenimiento y obtención de la materia prima.

Número de unidades de la vegetación silvestre y sitios antropogénicos donde hay manejo y presencia de las distintas variedades.

Número de personas que reconocen la presencia de la variedad en la vegetación silvestre.

Número de personas que reconocen la presencia de la variedad en milpas, ya sea como lindero o plantación.

Número de personas que reconocen la presencia de la variedad en espacios cercanos a la casahabitación. 